\title{
2. Die großen Raubzüge im 4. Jahrhundert
}

Isauri satis mali sunt et frequenter latrunculantur.

(Pereg. Aeth. XXIII 4)

Die größten isaurischen Raubzüge und Aufstände fanden nicht, wie es nahe läge, im krisenhaften 3. Jahrhundert statt, sondern nach der Konsolidierung des römischen Herrschaftssystems unter der Tetrarchie spätestens ab der zweiten Hälfte des 4 . und bis weit in das 5. Jahrhundert hinein. ${ }^{1}$ Vor der Erläuterung der Ursachen dieser Aufstände im folgenden Kapitel soll zunächst deren Ablauf dargelegt werden. Sie gipfelten um die Wende zum 5. Jahrhundert in einer Serie von Raubzügen, die sich über den gesamten östlichen Mittelmeerraum erstreckten.

\subsection{Die isaurischen Aufstände der zweiten Jahrhunderthälfte}

Die drei großen Isaurieraufstände in der zweiten Hälfte des 4. Jahrhunderts unter Constantius II. in den Jahren 354, 359 und 368 unter Valens schildert Ammianus Marcellinus in drei Passagen seines Geschichtswerkes, mit Sicherheit auf Grundlage militärischer Berichterstattung, zu der er wohl Zugang hatte. ${ }^{2}$ Zusätzlich zu diesen bei Ammian beschriebenen Konflikten und der Usurpation des Procopius 365 bis 366 gibt es noch Hinweise bei Eunap und Zosimus sowie bei Basilius von Caesarea, die einen weiteren, vierten größeren Aufstand im Jahr 375 unter Valens nahelegen. ${ }^{3}$ Weitere Unruhen in der ersten Jahrhunderthälfte deutet eine Rede des Himerius aus dem Jahr

1 Die Kirchenhistoriker überliefern für die erste Hälfte des 4. Jhs. keine isaurischen Aufstände. $\mathrm{Ob}$ Ammian in seinen diese Zeit behandelnde verlorenen Büchern solche erwähnt hat, ist ungewiß, vgl. SHAW (1990) 240, Anm. 114 u. III 2.2.

2 Amm. XIV 2. XIX 13. XXVII 9, 6 ff.; Ammian stammte aus einer griechischen Curialenfamilie aus Syrien, diente im Stabe des Heermeisters Ursicinus, 355 in Köln, 362/63 auf dem Perserzug Julians und lebte etwa 380-395 in Rom. Seine ,Res gestae" behandeln anknüpfend an Tacitus die Zeit von 98 bis 378. Erhalten ab Buch XIV, d. h. ab 353; vgl. Demandt (1965); Sabbah (1978); Matthews (1989); Barnes (1998). Zur Regierung des Constantius vgl. Otto SEECK, s.v. 'Constantius 4', RE IV 1 (1900), 1044 1094; PLRE I 226 s.n. 'Fl. Iul. Constantius 8'; Otto SEECK., s.v. 'Constantius 5', RE IV 1 (1900), 1094-1099; PLRE I 224f. s.n. 'Fl. Claudius Constantius Gallus 4'. Die Darstellung der Isaurierkämpfe durch ROUGÉ (1966) wird von HOPWOOD (1999b) als bloße explication du texte ohne Berücksichtigung der geographischen Gegebenheiten Isauriens kritisiert, doch sein Erklärungsansatz mit Betonung der Transhumanz als konstitutiv für das Widerstandsverhalten muß aufgrund fehlender Quellengrundlage und einem anachronistischen Ansatz als zu spekulativ angesehen werden, vgl. Kap. II.2.4. u. III.3.2.

3 LENSKI (1999b). 
341 an. $^{4}$ Zudem läßt die Formulierung Ammians, daß die Isaurier gewohnheitsmäßig der Räuberei frönten und sich wiederholt von den römischen Autoritäten befrieden ließen, eventuell auf die Schilderung weiterer Aufstände in den verlorenen, an Tacitus anknüpfenden Büchern wohl in der ersten Hälfte des 4. Jahrhunderts schließen. ${ }^{5}$

\subsubsection{Der große Aufstand des Jahres 354}

Im Jahr 354 wurde ganz Isaurien von einer weiträumigen Erhebung erfaßt, in deren Verlauf mehrere Legionen einbezogen waren und die Provinzhauptstadt Seleucia selbst unter Belagerung durch eine große Zahl isaurischer Banditen geriet. Was als kleiner Aufstand begonnen hatte, wuchs sich sehr rasch zu einem regelrechten Krieg (ad bella gravia promperunt) aus und konnte am Ende nur durch herbeigerufene weitere militärische Unterstützung beendet werden. Er wurde nach Ammians Schilderung offenbar durch das Fehlverhalten römischer Offizieller verursacht und reiht sich in seine Kritik an der Verwaltungspraxis unter Constantius II. und seinem in Antiochia residierenden Caesar Gallus ein (Buch XIV beginnt mit einer sehr negativen Charakteristik). Auch in Judäa gab es Unruhen, und in Syrien fielen plündernde Sarazenen ein. ${ }^{6}$

Ammian gibt vor dieser längeren Erzählung eine kurze Einführung, worin er den „Bandenkrieg“ der römischen Legionen gegen die Isaurier charakterisiert. Diese ließen es niemals zu einer offenen Feldschlacht kommen, in der sie den Legionen unterlegen gewesen wären, sondern wendeten, unter Zuhilfenahme ihres unzugänglichen Terrains, eine hit-and-run-Taktik an. Kurzen Attacken folgte der rasche Rückzug in die Berge. ${ }^{7}$ Der erste Satz Ammians in der Schilderung dieser Episode, ,die Isaurier, deren Gewohnheit es ist, oft befriedet zu werden und ebenso oft durch unerwartete

4 Himer. or. 25, Z. 88-98, freundlicher Hinweis von Harald VöLKER. Himerius preist Scylacius dafür, in Pisidien die Räuber besiegt zu haben. Dessen Amt als vicarius Asiae legt CTh XI 30, 22 nahe. Die in PLRE I 811, s.n. 'Scylacius 1' geäußerte Vermutung, daß Scylacius die Briganten in seiner Eigenschaft als proconsul Achaiae, mithin des Amtes, das er zum Zeitpunkt des Panegyricus ausübte, bekämpft haben soll, ergibt geographisch keinen Sinn, und Himerius rekurriert im Text auch auf die vergangenen Leistungen des Scylacius. BRANDT (1992) 170 bringt diesen Vorfall mit den Isauriern in Verbindung, vgl. LENSKI (1999a) 422 mit dem Argument, daß hier das pisidische Cremna attackiert wurde und daß der bei Amm. XXVII 9, 6 ff. geschilderte Musonius (s.u.) ebenfalls vicarius Asiae war.

5 Amm. XIV 2, 1 (s.u.); vgl. Shaw (1990) 240, Anm. 114.

6 Amm. XIV 1, 1 (Beginn). XIV 2 (Auswuchs); Jones (1964) I 116; Demandt (1989) 84.

7 Amm. XIV 2; Hellenkemper (1986) („Bandenkrieg“); SHaw (1990) 242 (,hit-and-run“). 
Raubzüge alles in Verwirrung zu bringen“, ${ }^{8}$ legt den Schluß nahe, daß er einmal mehr einer Episode in seinem Geschichtswerk einen allgemeinen Satz voranschickte, um ihn durch die dann folgende Geschichte zu illustrieren. Wie es sich auch in den hier besprochenen Passagen zeigt, neigt Ammian in seiner Darstellung eher zum Generalisieren, als daß er die historischen oder sozialen Besonderheiten herausarbeiten würde, eine Methode, die als Charakteristikum der römischen Historiographie überhaupt gilt.

Die unmittelbare Ursache der Rebellion war nach Ammian, daß einige isaurische Straßenräuber im Amphitheater der nördlich an Isaurien angrenzenden lykaonischen Metropolis Iconium, offenbar als plakative Strafmaßnahme, den wilden Tieren zum Fraß vorgeworfen worden waren. ${ }^{10}$ Dies sei, so Ammian, praeter morem gewesen. ${ }^{11}$ Unter dem Caesar Gallus waren blutige Zirkusspiele wieder an der Tagesordnung. ${ }^{12}$ Grund für die grausame Bestrafung wird eine Heimsuchung von Iconium oder seines Umlandes durch isaurische Banditen gewesen sein: Die ohne Zeitangabe in den Miracula der Heiligen Thekla erwähnte „Belagerung“ der Stadt durch Banditen aus dem Umland deutet darauf hin. ${ }^{13}$ Ein Grund für die Beschwerden der Isaurier könnte gewesen sein, daß nun einige Isaurier, die mit den Untaten nicht unmittelbar zu tun hatten, ersatzweise aufgegriffen und inhaftiert, schließlich ad bestias verurteilt wurden, nachdem sich die eigentlichen Banditen längst wieder in die Berge zurückgezogen hatten.

Die Aufständischen, von ihren heimatlichen Basen abgeschnitten und vom Hun ger geplagt, griffen zur Selbsthilfe und begannen, die an der Küste entlang fahrenden Getreideschiffe zu überfallen, wenn sie anlegten. ${ }^{14}$ Bei diesen Getreideschiffen kann

8 Amm. XIV 2, 1: Isauri, quibus est usitatum saepe pacari saepeque inopinis excursibus cuncta miscere.

9 Vgl. HopWOOD (1999b) 228 in Rekurs auf Erich AuERBACH, Mimesis. Dargestellte Wirklichkeit in der abendländischen Literatur, Tübingen-Basel 1946/91994, 53-77; SABBAH (1978) passim zu Ammians Darstellungsweise. Hopwood l.c. weist darauf hin, daß Ammian sich mit der Behauptung selbst widerspreche, die Isaurier seien durch Straflosigkeit noch ermutigt worden, da der Aufstand der Strafe erst folgte. Man könnte diese Einleitung allerdings auch auf das Ende bezogen sehen, denn letztendlich gingen die Isaurier aus dieser Episode straflos heraus, indem sie sich nach Eintreffen der Verstärkung der Truppen des comes Orientis Nebridius zerstreuten, s.u.

10 Zu Amm. XIV 2: RovgÉ (1966) 292-295; Syme (1968) 44f.; TIB 4 54; SAlmon (1997) 76f.; HoPWOOD (1999b) 228. Amm. l.c. bezeichnet Iconium irrtümlich als pisidische Stadt.

11 Die rechtliche Diskussion hierzu u. Kap. III.2.4.

12 Amm. XIV 7, 3; Iulian Misopog. 340 A; Otto SEECK, s.v. 'Constantius 5', RE IV 1 (1900), $1094-1099.1096 \mathrm{ff}$.

13 Mir. 6 mit Dagron (1978) 116, Anm. 2.

14 HOPWOOD (1999b) 228 verneint die Historizität dieser Schiffseroberungen und postuliert ihre Schilderung durch Amm. durch rein stilistische Gründe motiviert, aufgrund einer an- 
es sich nur um jene aus dem ägyptischen Alexandria nach Konstantinopel gehandelt haben; die Isaurier bedrohten damit die Versorgung der Hauptstadt, auch die Belieferung des isaurischen Hinterlandes über Seleucia und den Kalykadnos dürfte ebenfalls in Mitleidenschaft gezogen worden sein.

Daraufhin verlagerte sich die Küstenschiffahrt an die gegenüberliegende zypriotische Küste. Da sie nun unter ernsthafter Nahrungsmittelknappheit litten, begannen die Isaurier, in das nördlich angrenzende Lykaonien einzufallen und Straßensperren zu errichten. Die römischen Einheiten waren den Banditen im Gebirge unterlegen, doch in der Ebene konnten einige Scharmützel durch die Legionäre gewonnen werden, sie schlachteten die an Bewaffnung unterlegenen Isaurier „wie unnützes Vieh" ab. ${ }^{15}$ Es gelang dem Militär schließlich, sie aus dem ebeneren Lykaonien zu vertreiben.

Daraufhin wandten sie sich in die Richtung des westlich gelegenen Pamphylien. Am Grenzfluß Melas wurden sie von den isaurischen Legionen Isaura I, II und III gestellt, die in Side überwinterten. ${ }^{16}$ Nachdem sie den Fluß nicht überqueren konnten und sich quer durch Isaurien an den Nordhang des Taurus nach Laranda zurückgezogen und verschanzt hatten, gerieten sie bald wieder in Nahrungsmittelknappheit - ein logistisches Dilemma, welches die isaurischen Aufständischen mit den von außen ins Reich einfallenden Barbarenhorden teilten. ${ }^{17}$ Sie zogen nun wieder seewärts und ver-

genommenen literarischen Parallele zur bei Hdt. IV 110 beschriebenen Eroberung von Schiffen durch die Amazonen.

15 Amm. XIV 2, 7: pecudum ritu inertium trucidantur.

16 HopwOOD (1999b) 229 vermutet schon für 354 die Aufwertung der I Isaurica sagittaria zur Feldarmee und nimmt die unter Diocletian im nahen Colybrassus stationierte legio I Pontica als dritte isaurische Legion an, doch die I Isaurica wurde erst unter Valens mobilisiert, s.o.; vgl. LENSKI (1995) 138f. (1999b) 313, Anm. 21, und ein Verbleib der I Pontica bis zu diesem Zeitpunkt ist nicht sicher, s.o. Kap. III.1.9. Seyfarth Amm. (1968) 258, Anm. 31 bezweifelt unter Hinweis auf die Ungenauigkeit Ammians, daß es sich um mehrere Legionen gehandelt habe. Zur Funktion von Flüssen als Grenze mit diesem Beispiel vgl. NiCASIE (1998) 125, Anm. 17.

17 Hierzu vgl. die treffende Analyse durch NiCASIE (1998) 170f. mit Anm. 207, 212 (Isaurier): „It is a truism that logistic matters were of preeminent importance in ancient warfare. Generally, once barbarian invaders had forced an entry into Roman territory, they would have to fan out in order to obtain the necessary supplies. The small bands that were the result of this dispersion could be dealt with by comparatively small forces, but were difficult to locate and pin down; hence the importance of a close-knit network of observation posts and guarded roads. Because of this strategy, barbarian invaders sooner or later faced a dilemma: either to keep their forces dispersed, alleviating the procurement of supplies but increasing the risk of defeat in detail, or to concentrate their forces, which on the one hand improved their chance of successfully withstanding the Roman troops sent against them, but on the other seriously aggravated their logistic situation [...] the defence against Roman invasion collapsed because the barbarians were simply starved into submission or retreat or were defeated in detail." 
suchten vergeblich einen Angriff auf die westlich von Anemurium gelegene Festung Palae an der Südküste. Dieses Fort war angesichts der im Winter schwer passierbaren Nachschublinien zentrale Versorgungsstation für die isaurischen Legionen und somit von eminenter strategischer Bedeutung. ${ }^{18}$ Anschließend begannen die Isaurier, selbst die Metropolis Seleucia zu belagern, die von den drei isaurischen Legionen unter dem comes Isauriae Castricius verteidigt wurde. ${ }^{19} \mathrm{Da}$ die Isaurier nach Ammians Angabe zahlenmäßig diesen drei Legionen überlegen waren, muß ihre Zahl um die 5000 Mann betragen haben, wenn sich die Gesamtstärke der drei isaurischen Legionen bei einer angenommenen Mindest-Stärke der limitanei im Osten von 1000 Mann pro Legion zu diesem Zeitpunkt insgesamt auf mindestens 3 000, höchstens 4000 Mann belief. ${ }^{20}$

Die römischen Legionen, vor der Stadt schon zur Schlacht aufgestellt, zogen sich auf Befehl wieder zurück. Aus taktischen Überlegungen heraus wollte Castricius angesichts der zahlenmäßigen Überlegenheit der Isaurier kein Risiko eingehen und zog es vor, auf Verstärkung zu warten. ${ }^{21}$ Den Banditen gelang es jedoch, die Versor-

18 Amm. XIV 2, 13; Seyfarth Amm. (1968) 258, Anm. 35; De Jonge Amm. (1935) 115f.; heute Tahta Limanı, vgl. Hild / Hellenkemper (1986) 34f; TIB 5,1 s.v. 'Palaiai' 372; HOPWOOD (1999b) 229; NICASIE (1998) 149 mit Anm. 119 zur logistischen Bedeutung von Palae.

19 Amm. XIV 2, 15; Mir. 5 mit Dagron (1978) 116. Zu Castricius s.o. Kap. III.1.10; PLRE I 186 s.n. 'Castricius 1'; Lib. ep. 426; SEECK (1906) $103 f$.

20 Zur Sollstärke und Schätzungen der tatsächlichen Zahl der spätantiken römischen Legionen JONES (1964) II 680-683 mit III 210f., Anm. 174 (Belege aus Amm. u. Zos.). Seit Diocletian bis zur Abfassung der ND habe sie von 6000 auf 1000 pro Legion abgenommen, um das Jahr 359 bei der Belagerung von Amida befanden sich unter den 20000 Eingeschlossenen sieben allerdings stark dezimierte Legionen, Amm. XIX 2, 14; zur Zahl auch DEMANDT (1989) 256f. mit Anm. 1 ff.; zur Stelle DE JONGE Amm. (1935) 117 (1000 Mann). Gegen die Annahme von Jones (1964) II 682, der comes Isauriae hätte (offenbar für die zum Ende des 4. Jhs, in der ND or. XXIX 7.8 belegte Stärke von nur noch zwei Legionen) über eine Truppe von 6000 Mann verfügt, postuliert TREADGOLD (1995) 48. 51.63 realistischer bereits für diesen frühen Zeitpunkt eine Stärke von nur 2000 Mann; ebenso CoELLO (1996) 32: Zusammenstellung und Diskussion aller Quellen mit Tabelle. Proc. und Agathias nennen für Infanterie-Stadtgarnisonen, zu denen auch die isaurischen Legionen gehörten, für Justinians Kriege im 6. Jh. mehrmals eine Zahl von 1000, Belege bei NICASIE (1998) 72, Anm. 145, 74. 257.

21 Hopwood (1989a) 197 vermutet unter Verweis auf das Verhalten von Soldaten bei Kolonialkriegen in Südasien, daß die hier geschilderte Begierde zum Kampf eine Begierde nach Überlauf gewesen sein könnte. Doch ein derartiges Ereignis hätte der zum Moralisieren neigende Ammian kaum unerwähnt gelassen. Die vielen Erklärungen der Taktik mit der Betonung der securitas der Truppen legen nahe, daß die Quelle Ammians ein Rechtfertigungsbericht des Castricius gewesen sein könnte, so SABBAH (1978) 165 (,,rapport justificatif"), denn die vielfache Rechtfertigung des Rückzugs einer „kampferprobten Armee" 
gungsschiffe zu kapern, die über den Kalykadnos Seleucia und wohl auch das Hinterland bis hinauf nach Germanicopolis versorgten. ${ }^{22}$ Seleucia konnte schließlich durch das Eingreifen des comes Orientis Nebridius entsetzt werden. Dieser war als Zivilbeamter mit militärischer Vollmacht ausgestattet worden, denn der eigentlich zuständige magister militum per Orientem Ursicinus war zu diesem Zeitpunkt an der Perserfront beschäftigt. ${ }^{23}$ Sein Amtsbruder Honoratus (er wurde 359 der erste praefectus urbi Konstantinopels) stand zwar ebenfalls schon in der Nachbarprovinz Cilicia, ${ }^{24}$ doch die Isaurier konnten nicht kurzfristig eingekesselt werden, sie wichen angesichts der herannahenden militärischen Übermacht in die unwegsame Berglandschaft aus.

Da Nebridius sich angesichts der unruhigen Lage an der persischen Front nun auf keine größere und längere Verfolgung der Banditen einlassen konnte, zumal es taktisch sinnlos gewesen wäre, mit einer großen Armee zerstreute Flüchtige zu jagen, ist anzunehmen, daß die isaurischen Bergbewohner nach diesem militärischen Fiasko ersatzweise als Strafmaßnahme mit einem Handelsembargo belegt wurden: ein Brief des Libanius von 357 an seinen in Seleucia wohnenden Freund Acacius deutet darauf hin, daß das Styrax-Harz, eine isaurische Spezialität, offenbar nicht mehr auf dem Markt zu bekommen war. ${ }^{25}$ Zusätzlich ergriff die militärische Führung Präventivmaßnahmen zur Sicherung der Zugangswege: Das an der strategisch wichtigen StraBenverbindung durch den Taurus-Höhenzug zwischen Anemurium und Laranda gelegene Eirenopolis, wurde kurz darauf, zwischen 355 und 359, durch den comes

(Amm. XIV 2, 14: tresque legiones bellicis sudoribus induratae) in die sichere Stadt ist angesichts seiner sonstigen Kritik an der als feige und unmotiviert gezeichneten Armee auffallend, dazu vgl. DEMANDT (1965) 28, 55, 59. Nicht zutreffend ist daher die Annahme von HoPWOOD (1999a) 179, der die anfängliche Weigerung der Legionäre, gegen die Isaurier zu kämpfen, mit der nicht lange zurückliegenden persischen Katastrophe Julians erklärt.

22 Amm. XIV 2, 19; vgl. NiCASIE (1998) 149, Anm. 119.

23 Amm. XIV 2, 20; RougÉ (1966) 293, 303. Kurze Zeit darauf wurde Ursicinus von dem mißtrauischen Constantius II. nach Konstantinopel beordert, Amm. XIV 9, 2. 11, 2f,, vg1. PLRE I 985f. s.n. 'Vrsicinus 2'; zu Nebridius vgl. Lib. ep. 561. 1315; PLRE I 619 s.n. 'Nebridius 1'; SEECK (1906) 219 zu seiner Karriere; DE JONGE Amm. (1935) 118f.; Wilhelm ENSSLIN, s.v. 'Nebridius 2', RE Suppl. VII (1940) 549f.; RougÉ (1966) 302f. JONES (1964) III 16, Anm. 61 sieht Nebridius als Justizbamten an.

24 Lib. ep. 251. 386. 400; SEECK (1906) 179; Amm. XIV 1, 3. 7, 2 (Intrigen des Gallus gegen Honoratus); PLRE I 438f. s.n. 'Honoratus 2'.

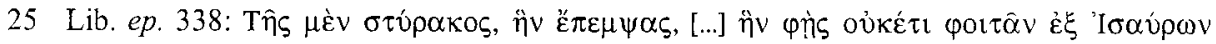

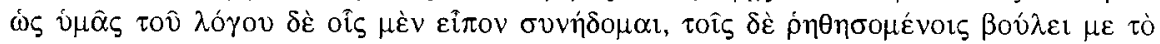
$\beta \lambda \alpha \sigma \varphi \eta \mu$ ov $\alpha \varphi \varepsilon \hat{\imath} v \alpha$ l. Zum Styrax s.o. Kap. III.2.4. Zu Acacius PLRE I 6f., s.n. 'Acacius 7’; SEECK (1906) 44. Rougé (1966) 293 vermutet dabei eine langfristige Isolierung der Stadt durch die Banditen, doch dies hätte bei Libanius einen deutlicheren Niederschlag gefunden. 
Isauriae Aurelius Iustus befestigt, um den Übergang am südlichen Arm des Kalykadnos von Anemurium aus ins Landesinnere zu sichern. ${ }^{26}$ Die Stadt mit dem programmatischen Namen war im 1. Jahrhundert n. Chr. nach den Aufständen der Kietai offenbar schon mit derselben Zielsetzung gegründet worden. ${ }^{27}$ Über diesen Paß waren die Isaurier wohl bei ihrem Angriff auf Palae gekommen.

Die hohe, vermutlich vierstellige Zahl der isaurischen Belagerer von Seleucia zeigt, daß sie in diesem Falle, wie schon zuvor bei dem Krieg um Cremna, die reinen Raubzüge die Schwelle zu einer militärischen Auseinandersetzung mit den römischen Legionen überschritten hatten. Doch der zweimalige Marsch des isaurischen Pulks quer in Nord-Süd-Richtung durch das Land läßt nicht auf ausgeklügelte Planung schließen. Die Isaurier waren durch Nahrungsknappheit gezwungen, größere Städte oder das Versorgungs-Fort in Palae anzugreifen. Beide Versuche mißlangen jedoch aufgrund der nicht vorhandenen militärischen Ausstattung. Daß die Isaurier mit diesem Aufstand jedoch einen größeren Plan in die Richtung einer etwaigen Unabhängigkeit von Rom verfolgten, ist zu bezweifeln. Die sich über ganz Isaurien erstreckenden Operationen lassen zwar auf Kommunikations- und Kommandostrukturen schließen, welche die lokale Ebene überschritten. Die kommunale Ordnung in Isaurien war durch diesen Vorfall zwar schwer erschüttert, doch gelang es den Isauriern nicht, einen eigenen, ausschließlich von ihnen kontrollierten Raum zu schaffen, wie ein Satz der Historia Augusta zuweilen interpretiert wird. ${ }^{28}$

\subsubsection{Frieden durch Verhandlungsgeschick 359}

Kurz nach der Befestigung von Eirenopolis entstanden dennoch erneut Unruhen in Isaurien, wohl weil die nach dem Aufstand von 354 ergriffenen wirtschaftlichen Sanktionen zu drückend geworden waren. Der mittlerweile zuständige comes Isauriae Bassidius Lauricius hatte aus der Fruchtlosigkeit der größeren Militäraktion fünf Jahre zuvor gelernt und konnte den Frieden eher durch Verhandlungen - vermutlich über isaurische Mittelsmänner, wie dies 368 über die Einwohner von Germanicopolis geschah - als durch militärische Gewalt erreichen. ${ }^{29}$ Ein Zeugnis seiner gleichwohl

26 Repertorium 82, 'Eir 1' = BM II 205, Nr. 231: murus aedi[ficatus est] Hirenopoli[tanorum civitati]. Zu Aurelius s.o. Kap. III.1.10. u. ArCE (1973); Martindale (1980) 487; MathISEN (1987) 13 (PLRE-Ergänzung).

27 S.o. Kap. III.1.6.

28 SHA trig. tyr. 26, 7; HellenKemPER (1986) passim; Shaw (1990) passim; zur Diskussion der behaupteten Quasi-Autonomie Isauriens s.u. III.3.2.2.

29 Amm. XIX 13; zu Lauricius Soc. h.e. II 39; Soz. h.e. IV 22, 2; Jul. Ep. 80; Lib. ep. 585; Epiphanius, adv. Haer. 73, 25, 3; PLRE I 497 s.n. 'Bassidius Lauricius'; s.o. Kap. III.1.10. SALMON (1997) 78 nennt die Vorgehensweise des Lauricius eine ,politique de pacifica- 
auch erfolgreichen militärischen Tätigkeit in dieser Provinz ist eine in eine Felswand an der Straße eingehauene Inschrift aus demselben Jahr 359. Die Straße gewährte im Sommer vom nördlich gelegenen Antiochia beim Dorf Çukur in der Nähe des heutigen Bucakkişla Zugang nach Germanicopolis, im Tal des nördlichen Quellarmes des Kalykadnos, und war somit von eminenter strategischer Bedeutung. Darin teilt Bassidius mit, daß er eine Festung erobert habe, die zum Schaden des Umlands lange Zeit von Banditen besetzt gewesen sei. Für die dauerhafte Sicherung des Friedens legte er eine Besatzung von Legionären hinein. ${ }^{30}$ Vermutlich handelt es sich bei dem castellum um einen befestigten Turm aus hellenistischer Zeit, der von den Römern dann wieder verstärkt wurde, wie es auch beim Turm in Diocaesarea der Fall war. Diese Türme waren seit hellenistischer Zeit im Besitz der städtischen Eliten, die so Kontrolle über die Landbewohner ausübten. ${ }^{31}$ Ebenso wie sein Vorgänger Aurelius Iustus hatte Lauricius so einen wichtigen Brückenkopf am Kalykadnos gesichert, hier den nördlichen Arm. Die römische Strategie bestand also darin, die unzugänglichen Höhenzüge durch Befestigungen in den langen, zugänglichen Talfluchten zu isolieren.

Ammian schließt seinen Bericht mit den Worten, daß die Verwaltung Isauriens durch die Regierungsweise des Bassidius Lauricius so gut war, daß es außer diesem kleinen Vorfall nichts Nennenswertes zu berichten gab. Er war mithin nur an den negativen Vorkommnissen interessiert, die er in seine Zeitkritik einfügen konnte. ${ }^{32}$ Die wahrscheinlich kurz nach diesen Ereignissen im Jahr 360 verfaßte Expositio totius mundi et gentium gibt sich zuversichtlich, daß die Römer die Isaurier letztlich unter Kontrolle gebracht hätten, wenngleich die Isaurier nach dieser Zeit die Phase der schlichten Raubzüge hinter sich gelassen und zu einem ernsthaften Krieg gegen die römische Beherrschung überzugehen geplant hätten, ${ }^{33}$ was Ammian ebenso mit seiner

tion". Der Kommentar von SEYFARTH ist an dieser Stelle irreführend: Lauricius habe die Raubzüge der Isaurier ,unterdrückt".

30 ILS $740=$ CIL III 6733: iussu dd. nn. Constantii triumfatoris | Augusti et Iuliani nob. Caesaris $\mid$ castellum diu ante a latronibus $\mid$ possessum et provinciis perniciosum $\mid$ Bassidius Lauricius v. c. com. et | praeses occupavit ad[q]ue ad perpe|tuam [q]ietis firmitatem militum | praesidio munitum Antiochiam | nuncupavit. Gefunden von DAvIS (1879) 367; vgl. TIB 5,1 s.v. 'Antiochia' 193. Zu Bassidius Lauricius vgl. Amm. XIX 13; Lib. ep. 585; Soc. h.e. II 39; Soz. h.e. IV 22 u. Kap. II.1.10.

31 Hopwood (1986) 346f. u. (1999b) 230.

32 Amm. XIX 13, 2: nihil accideret, quod animadversione dignum aestimaretur; vgl. DEMANDT (1965) 95. Diese Version ist der in der Budé-Ausgabe gegebenen Übersetzung „,qui justifiât une répression" vorzuziehen, so auch BARNes (1998) 92f. u. Anm. 74: „The assertion that nothing worthy of note happened in his province while he was governor can hardly be an innocent misapprehension."

33 Expositio 45: Isauria, quae viros fortes habere dicitur, et latrocinia aliquando facere conati sunt, magis vero et adversarii Romanorum esse voluerunt, sed non potuerunt invic- 
zu Beginn der Episode geäußerten Formulierung ad bella gravia proruperunt andeutet. Gegen die Realität dieser durch Iunior, den Autor der Expositio angedeuteten Möglichkeit, daß die Isaurier zu regelrechten „Gegnern“ Roms entwickeln könnten, spricht der Umstand, daß Kaiser Julian kurz darauf auf seinem Perserfeldzug die Regionen, welche von den Isauriern heimgesucht worden waren, völlig ungehindert durchqueren konnte. Auch die Korrespondenz des Libanius mit dem comes Isauriae Olympius Palladius gibt keinen Hinweis auf weitere bewaffnete Konflikte in den unmittelbar folgenden nächsten Jahren. ${ }^{34}$

\subsubsection{Die Usurpation des Procopius und ihre Folgen für Isaurien}

Zeitgleich mit den in diesem Kapitel vorgestellten großen isaurischen Aufständen ereignete sich im - zu dieser Zeit eigentlich stabilen - Herrschaftssystem des Ostreichs eine Usurpation. ${ }^{35}$ Diese Usurpation des Procopius und ihre Unterdrückung in den Jahren 365 bis 366 zog eine Schwächung der militärischen Besatzung in Isaurien nach sich, zumal gleichzeitig an der Perserfront gekämpft werden mußte. Procopius stammte selbst aus der Gegend, dem kilikischen Korykos, unweit von Seleucia, ${ }^{36}$ und besaß Güter bei Caesarea in Kappadokien, wohin er sich anfangs zurückzog. ${ }^{37}$ Procopius besaß zwar legitime Ansprüche auf das Kaisertum: er war mütterlicherseits verwandt mit Julian, da seine Mutter eine Schwester von Julians Mutter Basilina war, und hatte von Julian angeblich das Kaisertum versprochen bekommen. ${ }^{38}$ Er verzichtete aber nach dem Tod Julians im Perserkrieg und der Ernennung des Jovian auf die Macht und überführte Julians Leichnam von Tarsus nach Konstantinopel. Nachdem er dennoch nach Jovians kurzer Regierungszeit von Valentinian und Valens des Strebens nach dem Kaisertum verdächtigt wurde, trat er die Flucht nach vorne zur Usurpation an, verlor jedoch und wurde am 27. Mai 366 exekutiert. Höchstwahrscheinlich auf-

tum nomen vincere. Zum Datum vgl. RougÉ Expositio (1966) 27 ff. (1966) 294 mit Zweifel an der Verfasserschaft durch Iunior, anders DEMANDT (1989) 21.

34 Passio Arthemii 24 = Philostorg. h.e. VII 4c; Lib. ep. 837. 1238; SEECK (1906) 228; RoUGÉ (1966) 294; PLRE I 662 s.n. 'Olympius Palladius 18'.

35 Zum Ausnahmecharakter der Usurpation für das Ostreich Alan E. WARDMAN, 'Usurpers and internal conflicts in the 4th century A.D.' in: Historia 33 (1984), 220-237. 233f.; ELBERN (1984) passim; DEMANDT (1997).

36 Amm. XXVI 9, 11. 6, 1; PLRE I 742f. s.n. 'Procopius 4'; insgesamt Franz Josef WIEBE, Kaiser Valens und die heidnische Opposition, Bonn 1995. Sein gleichnamiger Verwandter war ein kilikischer Landbesitzer und galt als ein Wohltäter der Kilikier, Lib. ep. 194 (von 360); PLRE I 742 s.n. 'Procopius 2'.

37 Zos. IV 4, 3.

38 Seyfarth Amm. (1971) IV 308, Anm. 58 u. II 315, Anm. 186 zu XVII 14, 3. 
grund dieser Usurpation wurde die erste der drei isaurischen Legionen, die legio I Isaura sagittaria, eine wohl ehemalige Bogenschützentruppe, von einer Grenz- zu einer Feldlegion unter dem Kommando des magister militum per Orientem mobilisiert und stand somit als nunmehr pseudocomitatensische nicht mehr in Isaurien selbst zur Verfügung. ${ }^{39}$ Dies sollte sich zwei Jahre darauf beim im folgenden geschilderten dritten isaurischen Aufstand des 4. Jahrhunderts im Jahr 368 rächen, als die Isaurier einmal mehr mit knapper Not unter Zuhilfenahme von Truppen des Feldheeres besiegt werden konnten.

Bereits gegen Ende der Ende der Regierungszeit Constantins war es zu einer politischen Erschütterung in der Region gekommen, die wahrscheinlich zur wachsenden Unsicherheit in Isaurien beitrug. Im Jahr 334, noch zu Lebzeiten Constantins, rebellierte der zypriotische magister pecoris camelorum Calocaerus und beanspruchte die Herrschaft über die Insel. Sein Amt stellte eine Art Aufseher des staatlichen Transportwesens in quasi-militärischer Position dar, unmittelbar vor seiner Empörung dürfte er consularis der Insel gewesen sein. Der Halbbruder Constantins, Fl. Dalmatius, wurde mit der raschen Unterdrückung der Revolte beauftragt. ${ }^{40}$ Calocaerus wurde in der kilikischen Hauptstadt Tarsus exekutiert. Die Empörung griff auf die gegenüberliegende isaurische Küste über und dürfte auch das Hinterland betroffen haben. ${ }^{41}$

\subsubsection{Das Desaster des Musonius 368}

Neun Jahre nach diesem Vorfall jedoch plünderten isaurische Banditen in kleinen Gruppen wiederum Städte und Landsitze bis in die Nachbarprovinz Pamphylien hinein. ${ }^{42}$ Die wohl geringe Anzahl der Banditen sowie die militärische Schwächung der Provinz nach der Usurpation des Procopius kann die Erklärung dafür abgeben, warum mit der Bekämpfung der Banditen ein Zivilbeamter, der gelehrte und beliebte Historiker und vicarius Asiae Musonius mit Amtssitz in Sardis, der Provinzhauptstadt der

39 ND or. VII 56; LENSKI (1995) 138f. (1999b) 313, Anm. 21. Nach NiCASIE (1998) 191, Anm. 23 ist es nicht klar, ob derartige Legionsnamen Anachronismen aus der Entstehungszeit der Einheiten waren oder ihre gegenwärtige Bewaffnung oder Taktik reflektierten.

40 PLRE I s.n. 'Fl. Dalmatius 6', 240f.; voller Name P.Oxy. 1716.

41 Aur. Vict. Caes. 41, 11-12; Anon. Val. prior 6, 35; Hier. Chron. s.a. 334; Oros. VII 28, 30; Theoph. A.M. 5825; Cedr. I 519; vgl. Wilhelm Kroll, s.v. 'Kalokairos', RE X 2 (1919), 1757; zur Identifikation mit dem älteren Dalmatius, nicht dem gleichnamigen jungen Caesar, mit dem ihn Theoph. verwechselt vgl. PLRE I 177 s.n. 'Calocaerus'. Zum Titel magister pecoris camelorum vgl. DEMANDT (1970) 556.

42 Amm. XXVII 9, 6; DemandT (1989) 118. 
Lydia und der Diözese Asiana beauftragt wurde $:^{43}$ polizeiliche Aufgaben fielen zunächst unter das Kommado des zivilen vicarius, und Pamphylien gehörte bereits zur benachbarten Diözese Asiana. ${ }^{44}$ Auch in einem 25 Jahre zurückliegenden Vorfall um das Jahr 343 wurde ein vicarius Asiae namens Scylacius gegen wohl isaurische Räuber eingesetzt. ${ }^{45}$

Musonius heuerte vor Ort Diogmiten („Verfolger"), also leicht bewaffnete „Hilfssherriffs“ aus der lokalen Bevölkerung an. ${ }^{46}$ Diese waren jedoch aufgrund ihrer mangelnden Ausbildung keine ernsthaften Gegner für die Banditen: Er geriet mit seinem Trupp in einen Hinterhalt und kam um. Den genauen Ort gibt Ammian nicht an, doch der Untergang des Musonius dürfte sich nicht in Isaurien, sondern im benachbarten Pamphylien zugetragen haben, da die dortigen Truppen nicht erwähnt werden. Nach diesem Vorfall wurden „endlich“, so der kritische Ammian, „unsere Truppen aufgeboten" - wohl unter dem Kommando des aus Antiochia kommenden comes rei militaris Saturninus. ${ }^{47}$ Ammian kritisiert den zu spät erfolgten Einsatz des Militärs, denn nach den vorherigen Erfahrungen hätte man, wie er insinuiert, sogleich militärisch reagieren müssen. Doch dies scheint angesichts der bedrohlichen außen-

43 PLRE I 613 s.n. 'Musonius 2'.

44 Sein Landsmann und Zeitgenosse Eunap (349-nach 404) schildert ihn in frg. 43 als berühmten Gelehrten und überliefert mit frg. 43, 3 ein Nachruf-Epigramm. Zu Eun. vgl. Blockley (1981) 1-26; Buck (1988). (1998); Robert J. Penella, Greek Philosophers and Sophists in the fourth century A. D. Studies in Eunapius of Sardis, Leeds 1990. Sardis: Eun. frg. 43, 2 mit Blockley (1981) 1; Jones (1964) III 381, 387, 414 (Index) u. DEMANDT (1989) 249 zur Provinzeinteilung; RougÉ (1966) 303 zum vicarius Musonius; zum polizeilichen System der Poleis s.u. Kap. IV.3.1.2. Die Bemerkung von SHAw (1990) 243, daß Musonius' Qualifikation als Rhetor für dieses Amt unzureichend gewesen sei, ist insofern unzutreffend, als es seit der starken Einschränkung der Offizierslaufbahn für Senatoren durch Kaiser Gallienus (Aur. Vict. Caes. 33, 34) und der Trennung von militärischer und ziviler Laufbahn durch Constantin die gängige Praxis war, Gelehrte für die zivile Laufbahn einzustellen, wie es beispielsweise bei dem zeitgenössischen Rhetoren und späteren praefectus praetoriae Ausonius der Fall war; PLRE I 140f. s.n. 'Decimius Magnus Ausonius 7'; DEMANDT (1989) 257. 277. Die Zivilbeamten in der Provinzialverwaltung mußten noch die klassische Rhetorenausbildung nachweisen, vgl. TINNEFELD (1977) 107116. Daß Musonius ein berühmter Rhetor war, bevor er sein Amt antrat, ist also nichts Ungewöhnliches.

45 Himer. or. 25, Z. 88-98; s.o. - Die Überschriften zu Himer. or. 20.39 sind dem Musonius zugeschrieben, der dann jedoch gegen Ammians Zeugnis vicarius Macedoniae gewesen sein müßte, was nicht wahrscheinlich ist.

46 Amm. XXVII 9, 6: adhibitis semermibus paucis, quos Diogmitas appellant. Zur Ausführlichen Diskussion der Diogmiten s.u. Kap. IV.3.1.2.

47 Amm. XXVII 9, 7: excitae tandem copiae contruserunt. Basil. ep. 132 nennt ihn comes rei militaris in Antiochia; Mir. 13; PLRE I 807f. s.n. 'Saturninus 10'; zur Identifikation des in

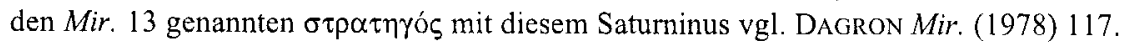


politischen Situation nicht ohne weiteres möglich gewesen zu sein. An eine Abordnung des comes Isauriae, zu dieser Zeit höchstwahrscheinlich Flavius Uranius, nach Pamphylien, war wohl angesichts der unsicheren Lage in Isaurien selbst nicht zu denken, und die mobile Feldarmee war in einem Krieg gegen die Goten an der Donau gebunden. ${ }^{48}$ Somit erwies sich das Gotenproblem erneut als einer raschen Ordnung der isaurischen Verhältnisse hinderlich.

Es gelang den römischen Truppen wiederum nicht, die vollständige Kontrolle über das Gebiet zu erringen. Auf Vermittlung der Bewohner von Germanicopolis wurde nun ein Waffenstillstand wie mit äußeren Feinden geschlossen, die Aufrührer stellten Geiseln. ${ }^{49}$ Das Zentrum des isaurischen Unruheherdes befand sich infolgedessen vermutlich in der Nähe von Germanicopolis. Nordwestlich der Dekapolis wird die Bergwelt sehr schroff und unzugänglich. ${ }^{50} \mathrm{Da}$ nur die Isaurier und nicht auch die Römer Geiseln stellten, verdeutlicht jedoch, daß dieser Verhandlungsfriede nicht aus einer klaren militärischen Pattsituation hervorging, weshalb eine Quasi-Autonomie für Isaurien keine korrekte Bezeichnung sein kann. ${ }^{51}$ Germanicopolis, die von Tiberius gegründete Veteranenkolonie, war mittlerweile die führende Stadt in der isaurischen Dekapolis, und ihre Führungsschicht verfügte offenbar über einen nicht geringen Einfluß auf die übrigen Bergbewohner. Das Zentrum Isauriens war nun von Isaura Nova in das fruchtbare Kalykadnostal gewandert. Ihre strategische Lage an der wichtigsten Straßenkreuzung im isaurischen Kernland, der Verbindung in alle Richtungen des Hochtaurus, versetzte die Stadt in eine entscheidende Position. Das Beziehungsgeflecht zwischen Stadt und Land dürfte sich vor allem auf den Wollhandel gestützt haben und scheint auch sonst Vermittlung und Rechtsprechung einbezogen zu haben. ${ }^{52}$

Der oben zitierte Satz Ammians, daß die Isaurier sich gewohnheitsmäßig befrieden ließen und ebenso oft wieder vertragsbrüchig würden, ${ }^{53}$ deutet darauf hin, daß dieser Vertrag nicht der erste zwischen der römischen staatlichen Autorität und auf-

48 Fl. Uranius befestigte Corasium als neue Hafenstadt, vgl, PLRE I 982 s.n. 'Fl. Vranius 5'; MAMA III 102-107. Die Arbeiten dürften die isaurischen Legionen ebenfalls gebunden haben, s.u. Zu den Gotenkriegen des Valens Amm. XXVII 5, 5; Wanke (1990) 84-110; HEATHER (1991) 116-121; LENSKI (1995) 206-264. (1999b) $311 \mathrm{f}$.

49 Amm. XXVII 9, 7; vgl. TIB 5, 37; HopWOOd (1986) 350f. u. (1999b) $231 \mathrm{f}$.

50 Vgl. HellenkEMPER (1986) 630. Die isaurischen „Klimata“, die er am Oberlauf des Kalykadnos lokalisiert, befanden sich jedoch eher im westlich gelegenen Tiefland, s.o. Kap. II.3.

51 Zur Diskussion s.u. im Text u. Kap. III.3.1.4.

52 MatTHEWs (1989) 365; HopWOOD (1986) 350f., (1991) 306 u. (1999b) 231f; TOMASCHITZ (1998); s.o. Kap. III.2.4. Hopwood (1999b) 232 vermutet, daß sie den Banditen auf ihren befestigten Gütern gleichzeitig Zuflucht verschafften und sie dadurch auch kontrollierten.

53 Amm. XIV 2, 1. SEYFaRTH l.c. übersetzt mit ,unterwerfen“. 
ständischen Isauriern war, worauf auch die oben geschilderte Episode unter der Regierung des Bassidius Lauricius hindeutet. Die Praxis der Vergeiselung war dabei üblich. Die Miracula der Thekla erwähnen eine sozial hoch stehende Geisel: Im Wallfahrtsheiligtum der Heiligen bei Seleucia wurde eine Frau aus einer Adelsfamilie der Ketis (also der Isaurier) ${ }^{54}$ namens Bassiane festgehalten. Dabei ist ein Zusammenhang mit dem hier geschilderten Ereignis denkbar, da sich die Episode laut Schilderung eine Generation vor der Abfassungszeit der Vita zugetragen hatte. ${ }^{55}$ Auch diese Vergeiselung deutet eher darauf hin, daß eine Quasi-Unabhängigkeit des isaurischen Kernlandes von Valens keineswegs anerkannt worden wäre. ${ }^{56}$

An Ammians Schilderungen fällt auf, daß er keinen einzigen der an den drei Aufständen beteiligten Isaurier oder gar einen Anführer beim Namen nennt. Dabei hat es zweifellos und nach dem erwähnten Zeugnis der Miracula eine deutliche Schichtung innerhalb der isaurischen Gesellschaft gegeben, sonst wäre jegliche Vergeiselung gar nicht erst erwogen worden. ${ }^{57}$ Doch sozial zu differenzieren oder gar die Ursachen der isaurischen Aufstände genauer zu erläutern war Ammians Sache nicht, seine Intention war vielmehr, die Aufstände als Krisensymptom im Rahmen seiner Zeitkritik zu demonstrieren. ${ }^{58}$

\subsubsection{Die Unruhen von 375: Eine Verbindung zur Schlacht von Adrianopel?}

Unter Valens entstanden im Jahr 375 erneut Unruhen in Isaurien, welche durch die stärkeren Militärmaßnahmen wohl auch indirekt verantwortlich für die schwache Verfassung des Ostheeres bei der Schlacht von Adrianopel gegen die Goten am 9. August 378 waren, so die plausible Rekonstruktion von Noel LeNSKI ${ }^{59}$ Zwar fällt

54 Die Ketis wird in der Spätantike zu Isaurien gezählt, TIB 5,1 301 s.v. 'Kētis'; s.o. Kap. II. 4 .

55 Mir. 19; vgl. Dagron Mir. (1978) 118.

56 So aber die Annahme von Rougé (1966) 295; SHAw (1990) passim u. (1984) 42: „Perhaps the best-documented long-term case of this type of banditry [quasi-state status] in the Roman empire is that found in Isauria."

57 Vgl. MatThews (1989) 365.

58 DEMANDT (1965) 28.

59 Die genaue Datierung des Aufstands auf das Jahr 375 wird durch Mir. 13 ermöglicht,

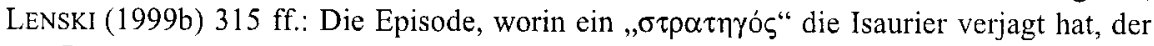
von Dagron Mir. (1978) 117 mit Saturninus identifiziert wurde (s.o.), bildet einen terminus ante quem zu 377, als dieser von Valens gegen die auf Reichsboden angesiedelten, revoltierenden Hunnen eingesetzt wurde, Amm. XXXI 8, 3. Zwei Grabinschriften aus Seleucia, Repertorium 353, 'Sel 29' = CIG 9207 u. Repertorium 351, 'Sel 20' = CIG 9230, verweisen auf zwei Soldaten der sagittarii dominici, einer Bogenschützen-Einheit, die von Valens aufgestellt wurde, ND or. VI 56, und deren tribunus Barzimeres in den Gefechten 
auf, daß Ammian diesen Vorfall nicht erwähnt, obwohl er die drei vorausgegangenen Aufstände ausführlich geschildert hat und seine Darstellung mit der Schlacht von Adrianopel schließt. Doch dies muß nicht unbedingt gegen die Historizität dieses Vorfalls sprechen, denn er unterschlägt auch andere kleinere militärische Konflikte, so einen gotischen Bürgerkrieg um 372 und einen Sarazenenaufstand um 377/378. Dies dürfte eine stilistische Maßnahme Ammians sein, um die Hinleitung seiner Erzählung auf die Klimax seines Werkes, die große Schlacht von Adrianopel, nicht zu stören. $^{60}$

Die Autoren Eunap und Zosimus sprechen von ,zahlreichen feindlichen Überfällen“ zu dieser Zeit. Zosimus schließt die Bemerkung an, daß Valens vor allem durch die Isaurier bedrängt wurde. Er erwähnt weiter, daß der Kaiser zum Zeitpunkt des Aussendens einer - „wie er meinte, ausreichenden“ - Streitmacht gegen die Isaurier (Zosimus deutet also an, daß gerade dies nicht der Fall war) sich in Antiochia aufgehalten habe. Er bringt diese Ereignisse unmittelbar vor der Schilderung der Schlacht von Adrianopel und legt damit eine Verbindung zwischen Isaurieraufstand von 375 und der Katastrophe nahe. ${ }^{61}$

Die Isaurier jedoch flüchteten, so Zosimus, mit ihrer gesamten Beute einmal mehr in die wildesten Teile des Gebirges, ohne daß die Soldaten (aus „Nachlässigkeit", wie

vor der Schlacht bei Adrianopel bei Amm. XXXI 8, 9 als einer der Gefolgsleute des Saturninus in Thrakien erwähnt ist, PLRE I 148 s.n. 'Barzimeres'.

60 Soc. h.e. IV 33, 1-4. 36; Soz. h.e. VI 38; Theodoret h.e. IV 23; Vermutung von LENSKI (1999b) 314.

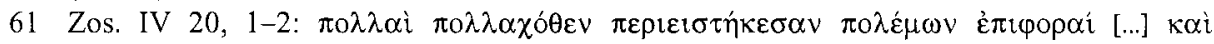

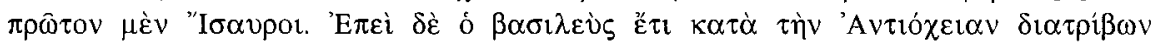

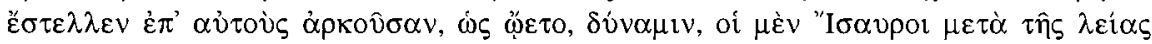

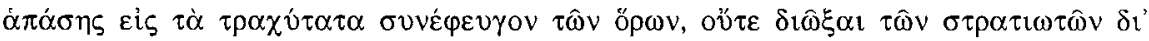

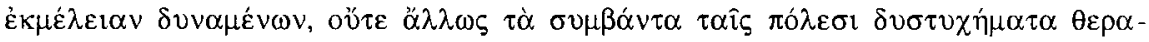
$\pi \varepsilon \hat{\sigma} \sigma \alpha$. Vgl. auch Eun. frg. 43, 4 mit BloCKLEY (1983) 141, Anm. 97; Basil. ep. 200. 215. 217. Santos Yanguas (1977) 367; Paschoud Zos. (1979) 371f. und Rebenich Zos. (1990) 340f., Anm 41 glaubten, daß Eunap, gefolgt von Zosimus, die Revolte von 368 auf 375 fehldatiert habe, weil sie bei Ammian keine Erwähnung finde. Dagegen meint BlocKLey (1983) 141, Anm. 97 im Verweis auf Eun. l.c., Zosimus habe die beiden Aufstände absichtlich an derselben Stelle beschrieben, denn er verweist ja expressis verbis darauf, ebenso CoYNe Priscian (1991) 80f. RougÉ (1966) 295f. datiert später auf 376/377, gefolgt von Minor (1979) 122f.; TIB 5,1 37. DAGRON Mir. (1978) 116 ff. vermutet einen längeren Aufstand, der sich von 368 bis 377 hinzog; HoPwood bietet in verschiedenen Artikeln, (1986) 344. (1989) 191. (1999b) 232, verschiedene Daten an. Gegen die Interpretation von PASCHOUd l.c. führt LENSKI (1999b) 314f. an, daß Valens vor 370 sich nachweislich nicht in Antiochia aufgehalten hat und daß bei Zosimus eine rasche Reaktion des Kaisers auf den Aufstand beschrieben wird, während das Militär beim Aufstand von 368 nach der Schilderung von Ammian nach dem Untergang des Musonius auf sich warten ließ. 
Zosimus moralisierend-kritisch anmerkt, in Wahrheit wohl wiederum eher aus taktischer Überlegung) nachzusetzen oder sonstwie den Übeln, welche die Städte getroffen hatten, abzuhelfen vermochten. Wiederum also wenden die Isaurier ihre schon sattsam aus den ammianischen Schilderungen bekannte Taktik des raschen Zuschlagens und des ebenso raschen Rückzugs an.

Des weiteren erwähnt Basilius von Caesarea in seinen zu dieser Zeit entstandenen Briefen zahlreiche Attacken von Banditen. Diese Banditen wurden in der bisherigen Forschung mit dem Aufstand der Goten in Thrakien in Zusammenhang gebracht, ${ }^{62}$ doch die Goten agierten westlich des Bosporus und nicht in Anatolien, denn Basilius bezog sich in seiner an den Presbyter Dorotheus aus Antiochia im Herbst 375 gerichteten Warnung auf die Landstrecke zwischen Antiochia und Konstantinopel, welche durch das Taurusgebirge führen konnte, so LENSKI. Dorotheus wird vor einer geplanten Reise nach Rom gewarnt, da die Route in Anatolien ,voller Feinde" sei. ${ }^{63}$

Gegen die Ansicht von SHAw, der annimmt, daß die Isaurier in ihrem Brigantentum und ihrem Widerstand gegen die römische Autorität durch alle Zeiten hindurch konstant blieben, zeigen die Quellen also, daß die Raubzüge im dritten Jahrhundert stark zunahmen, bis zu dem Punkt, als die isaurischen Machthaber die römische Politik im 5. Jahrhundert dominierten. Vor allem den isaurischen Unruhen von 375 kommt eine fatale Rolle beim Niedergang der römischen Abwehr gegen gotische Truppen zu: Sie beeinflußten die Bemühungen des Valens, die sich ab Herbst 376, nach den Ereignissen um den mißglückten Donauübergang ausweitende gotische Revolte einzudämmen, indem sie Truppen und Logistik abzogen. ${ }^{64}$ Valens ließ im Zeitraum zwischen 375 und 376 die Straße von Konstantinopel nach Caesarea erneuern, offensichtlich um den Truppentransport gerade nach Isaurien zu erleichtern, wie eine Serie von Meilensteinen aus dieser Zeit nahelegt. ${ }^{65}$

62 Holl (1904) 21 trennt die bei Basil. ep. 217, 81 genannten Barbareneinfall, der nach dessen Aussage die Rückkehr vieler zum Heidentum zur Folge hatte, von den isaurischen Raubzügen und legt eine gotische Invasion nahe: „Leider sagt weder Basilius, noch, so viel ich weiß, eine andere Quelle, wer genauer die $\beta \alpha ́ p \beta \alpha \rho p$ o gewesen sind"; für einen Bezug auf die Goten CourtonNe, Basil. (1957-1966) II 207, Anm. 1; Hauschild, Basil. (19731993) III 186f., Anm. 15. 18.

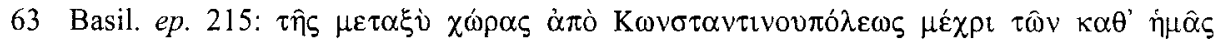

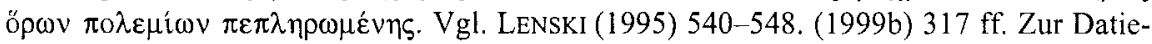
rung der Briefe Friedrich Loofs, Eustathius von Sebaste und die Chronologie der BasiliusBriefe. Halle 1898; Paul J. FEDwICK, 'A Revised Provisional Chronology of the Life and Works of Basil of Caesarea' in: Ders. (Hrsg.), The Church and Charisma of Leadership in Basil of Caesarea (= Pontifical Institute of Medieval Studies 45), Toronto 1979, 133-155.

64 So das Fazit von LeNSKI (1999b) 325.

65 FRENCH (1988) Nr. 637, 639, 643, 648-651, 655, 660; LENSKI (1999a) 424 
Eine weitere kurze Bemerkung Ammians läßt auf einen kleineren Konflikt wohl zur selben Zeit mit isaurischen Banditen schließen: Der Tribun Aliso, ein Kriegsheld seit der Einnahme von Kyzikos durch den Usurpator Procopius, fand einige Jahre nach diesem von Ammian im Jahr 366 geschilderten Ereignis in Isaurien, wohl während einer Patrouille, unter den Streichen einer Räuberbande den Tod. Möglicherweise provozierte die korrupte Amtsführung des damaligen comes Isauriae Natalis einen isaurischen Aufstand, der von Alisos kleiner Truppe niedergeschlagen werden sollte. ${ }^{66}$

Zwei der drei kanonischen, Regeln für die Gemeinden formulierenden Antwortbriefe des Basilius an Amphilochius zeugen von der weit verbreiteten Unsicherheit in der Gegend: Darin werden Priester ermahnt, sich nicht an die Spitze der zur Gegenwehr gegen Räuberbanden ausziehenden Bewohner zu stellen und die Banditen präventiv zu ermorden, was offensichtlich eine weitverbreitete Praxis war. Weiterhin sind Mädchenraub und Totschlag thematisiert. ${ }^{67}$ Das Militär wurde in Reaktion auf diese Situation aufgestockt: Von der Verschickung frischer Rekruten zeugt ein weiterer Basiliusbrief. Einer seiner Kuriere namens Meletius war ein Offizier, der solche Rekruten nach Iconium zu bringen hatte. ${ }^{68}$ Wie lange diese Situation verstärkter Unsicherheit andauerte, ist nicht zu ermitteln. ${ }^{69}$ Der comes rei militaris Saturninus blieb jedenfalls bis zum Jahr 377 in Isaurien, wo er sich in mehreren kleinen Scharmützeln engagierte. ${ }^{70} \mathrm{Da}$ sich die Unruhen auch über die folgenden Jahre hinzogen, kann davon ausgegangen werden, daß die Isaurier die Verteidigungskraft des Ostreichs am Vorabend der Schlacht von Adrianopel am 9. August 378 erheblich schwächten. ${ }^{71}$

66 Amm. XXVI 8, 10: in Isauria oppetit vastatoria manu confossus; PLRE I 45 s.n. 'Aliso'; Natalis: CTh IX 27, $3=C J$ IX 27, I von 382; vgl. LensKI (1999a) 425.

67 Basil. ep. (188). 199. 217; LENSKI (1999b) 318. Zur Definition der canones vgl. HaUSCHILD Basil. II (1973) 173, Anm. 209: Sie entwickelten sich im 4. Jh. von Regeln der Bußpraxis zu allgemeinen kirchlichen Rechtssätzen, vgl. auch u. Kap. III.3.1.2. zu den canones des Basilius.

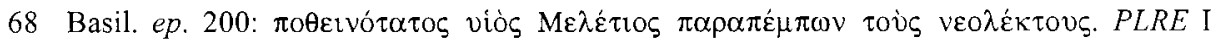
594, s.n. 'Meletius 3'; LeNSKI (1999b) 318.

69 Basil. ep. 232 aus dem Frühjahr 376 an Amphilochius, den LENSKI (1999b) 320 auf ein Ende des Konflikts zu diesem Zeitpunkt verweisen läßt, kann nicht herangezogen werden, denn die erwähnten „gewissen Unruhen“ beziehen sich auf die Gefangennahme und Flucht des Gregor von Nyssa und nicht auf die mit den Isauriern zu identifizierenden „Barbaren“; hierzu Hauschild Basil. (1993) 204, Anm. 184. 197, Anm. 126.

70 Amm. XXXI 8, 9; LENSKI (1999b) 316. Nach Mir. 13 wurde ihm einmal ein Hinterhalt gelegt, vor dem er jedoch durch die heilige Thekla gewarnt wurde, so daß die Isaurier allesamt niedergehauen werden konnten.

71 LENSKI (1999b) 325: „The Isaurian conflict of 375 probably played an important role in triggering the series of setbacks which led to the disaster at Adrianople". Zur Vorgeschichte Eun. frg. 42 (im Vergleich zur Darstellung des Amm. einseitig, vgl. BLOCKLEY (1983) 
Theodosius, der von Gratian nach dem Tod des Valens in der Schlacht eingesetzte Ostkaiser, mußte das Reich wieder konsolidieren; die Goten fielen ungehindert in die Balkanprovinzen ein. Auch in Isaurien lag die Ordnung weiterhin danieder: Der isaurische Bandit Balbinus attackierte wohl im Jahr 380 die isaurischen und kilikischen Städte Anazarbus, Eirenopolis und Kastabala. Er wurde von dem $\sigma \tau p \alpha \tau \eta \gamma o ́ \varsigma$ (wohl: comes rei militaris) Rufus gefangengenommen und getötet. ${ }^{72}$ Zosimus gibt einen Hinweis darauf, daß die Einwohner Pamphyliens sich gegen Ende des 4. Jahrhunderts schon an zahlreiche Überfälle aus Isaurien gewöhnt hatten, weshalb sie plündernde Goten unter Tribigild im Jahr 399 zurückwerfen konnten. ${ }^{73}$

Mit dem Frieden vom 3. Oktober 382 wurden die Goten als Föderaten anerkannt: sie siedelten zwar auf dem Reichsboden, waren jedoch ihren eigenen Führern unter-

140, Anm. 92) und der Schlacht selbst Amm. XXXI; SEECK (1921) V 466; HoffmanN (1969) 440 ff.; Matthews (1989) 296 ff.; Demandt (1989) 123; Wanke (1990) 198-219 (Kriegsrat, Gesandtschaften, Schlachtverlauf); Michael P. SPEIDEL, 'Sebastian's strike force at Adrianople' in: Klio 78 (1996), 434 437 (Verbindung von Eun. frg. 44, 5 mit Amm. XXXI); LENSKI (1997) passim zur zeitgenössischen Reaktion - Zos. IV 21 ff. mißt der Katastrophe keine providentielle Bedeutung zu, anders Lib. or. 24; Rufin. h.e. XI 13; Analyse der Schlacht durch NICASIE (1998) 233-256, der die Schlacht etwas gewagt als taktischen Fehler im Rahmen einer erfolgreichen Strategie einordnet. Letztlich habe die Entwicklung mit dem Gotenfoedus des Theodosius I. im Jahr 382 den ursprïnglich von Valens angestrebten Verlauf in Richtung einer gesteuerten Integration der Goten genommen; die Schlacht selbst habe mithin keine historische Bedeutung

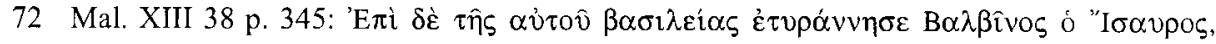

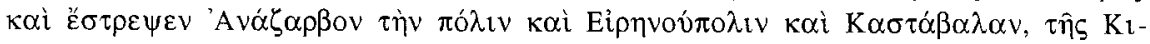

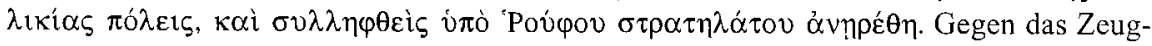
nis des Mal. spricht sich Brian CROKE, 'The Context and Date of Priscus Fragment 6' in: Classical Philology 78 (1983) 297-308 für eine Datierung in die Regierungszeit von Theodosius II. aus, gefolgt von ZUCKERMAN (1994) 180. Zwar ist unter diesem Theodosius auch ein Rufus als comes (rei militaris?) für das Jahr 448 in Antiocheia bezeugt, vgl. PLRE II 209 s.n. 'Balbinus'. I 784 s.n. 'Rufus 3'. II 958f., s.n. 'Rufus 1'. Jedoch ist die Stelle bei Const. Porph. in den Exc. de insid. 160, 17 ff. mit ǒ $\tau$ غ̇ $\pi \grave{i} \Theta \varepsilon \circ \delta \circ \sigma i o v ~ \tau o \hat{v}$

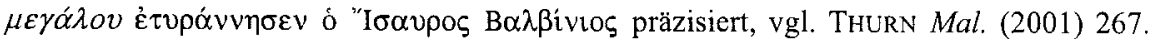
Abgesehen davon war der Rufus von 448 ein Gefolgsmann des ersten Zeno, und es ist wenig wahrscheinlich, daß zu einem Zeitpunkt von großer Machtfülle eines anderen Isauriers sich ein Bandit erhoben hat. Die Geschichte dieses räuberischen Aufstands fügt sich insgesamt eher in den Kontext des ausgehenden 4. Jahrhunderts ein. TIB 5,1 145 verweisen zudem auf einen möglichen Zusammenhang des Balbinus-Aufstands mit der Reparatur der Mauer in Anemurium. Die 16 Jahre später erfolgte Reparatur der Mauer von Kastabala, der anderen von Balbinus attackierten Stadt, unter Aufsicht des magister militum per Orientem Simplicius; PLRE II 1013f., s.n. 'Simplicius 2' dagegen steht wohl eher im Zusammenhang mit den großen Unruhen aus diesem Jahr und im Schatten der heraufziehenden Gainaskrise; vgl, auch TIB 5,1 38; HILD (1993) 222; LENSKI (1999a) 424. Zu Gainas s.u.

73 Zos. V 14; vgl. Hopwood (1986) 348 u. Text unten. 
geben. Damit wurde ihnen ein „Schwebezustand zwischen Reichszugehörigkeit und Autonomie" zugestanden. ${ }^{74}$ Der neue Kaiser war dabei offenbar gewillt, mit dem Goten- auch das Isaurierproblem auf die gleiche Weise zu lösen: Die erste von Theodosius in Dienst gestellte rein isaurische Truppe der Felices Theodosiani wurde wohl im Rahmen eines (nicht in den Quellen dokumentierten) Friedensvertrags, der einem weiteren isaurischen Aufstand folgte, aufgestellt. In einem Panegyricus auf den Kaiser aus dem Jahr 389, der einen vorangegangenen Aufstand im Taurusgebirge nennt, ist höchstwahrscheinlich eben diese Truppe gemeint. ${ }^{75}$

Die isaurischen Kämpfer wurden also gleich den gotischen behandelt. Dieser Umstand zeigt sich an einer weiteren Maßnahme des Kaisers: Um die Gefahr von Konflikten, die durch zu große Ansammlungen bewaffneter Goten generiert wurden, wie jenem im Vorfeld der Schlacht von Adrianopel, von vornherein auszuschließen, wurden einzelne in Thrakien stationierte gotische Verbände im Austausch mit dortigen Truppenteilen nach Ägypten verschickt. ${ }^{76}$ Da um das Jahr 444 ein numerus Isaurorum in Alexandria bezeugt ist, liegt es nahe, auch die Entstehung dieser Truppe durch die Maßnahme des Theodosius zu erklären. ${ }^{77}$

\subsection{Die Befestigung von Städten und Gehöften}

Die ständigen Unruhen in Isaurien machten nicht nur eine stärkere Militärpräsenz nötig, sondern führten in dieser Zeit auch zu einer Befestigung von Städten und kleineren Gehöften. In der zweiten Hälfte des 4. Jahrhunderts ist die Befestigung der Städte Eirenopolis, ${ }^{78}$ Corasium und Anemurium belegt. Die vom comes Isauriae Flavius Uranius zwischen 367 und 375 im Kalykadnosdelta vor Seleucia neu gegründete Hafenstadt Corasium (Susanoğlu) bekam gleich beim Baubeginn eine Wehrmau-

74 Demandt (1989) 126f. zu Zos. IV 30; zum positiven Bild des Theodosius I. in den Quellen vgl. Jörg ERNESTI, Princeps christianus und Kaiser aller Römer, Paderborn-München 1998.

75 Felices Theodosiani Isauri: ND or. V 66; Panegyricus des Pacatus: Paneg. Lat. II (XIX) 33, 4: hos [...] rigens Taurus [...] effuderat. Vgl. LieBeschuetz (1990) 30; Lenski (1999a) $424 \mathrm{f}$.

76 Zos. IV 30, 2. Zum Einsatz gotischer Truppen in Ägypten vgl. HoffmAnN (1969) 230 mit Anm. 213. 235f. mit Anm. 270. 237f. mit Anm. 293. 460f. mit Anm. 240 ff., der Datierung nach 379, spätestens Anfang 380, da bei den Kämpfen in Makedonien gegen die Goten bereits ägyptische Einheiten mitkämpften, vgl. Zos. IV 31, 2.

77 Dagegen vermutet LENSKI (1999a) 425 die Bildung dieser Truppe aus einem foedus nach dem Aufstand von 441: Marc. Com. s.a. 441; Prisc. frg. 10 kann auch auf 447 bezogen werden, vgl. Croke (1983c); Theod. h.r. X. Zum Entstehen der isaurischen Truppen und zum alexandrinischen numerus Isaurorum $\mathrm{s}$. auch u. Kap. V.1.1.

78 S.o. Kap. IV.2.2.1. 
er, da sie entfernt von den übrigen Siedlungen war. Der Neubau des Hafens war wohl durch die schlechte Landverbindung zwischen der Provinzhaupstadt und ihrem Hafen motiviert, denn ein im Südosten Seleucias gelegener Fels, durch den nur ein schmaler und durch etwaige Feinde leicht zu sperrender Weg führte, liegt kurz vor dem TheklaHeiligtum auf der Verbindungslinie zwischen Hafen und Stadt und bildet noch heute die Verbindung zwischen Stadt und Ruinenplatz. ${ }^{79}$ Zudem ist eine zunehmende Versandung des Hafens im Kalykadnosdelta denkbar. Die Ereignisse von 354, als Seleucia von den isaurischen Banditen eingeschlossen war, haben diese prekäre Situation gezeigt. Der neue Hafen garantierte durch seine Befestigung ein weit höheres $\mathrm{Ma} ß$ an Sicherheit. $^{80}$

\subsubsection{Die Verstärkung der Mauer von Anemurium}

Im Jahr 382 ließ der comes Isauriae Matronianus, Nachfolger des Natalis, durch den Kommandanten der pseudocomitatensischen legio I Armeniaca, die dem magister militum per Orientem unterstand und als Verstärkung herbeigerufen worden war, ${ }^{81}$ die

79 Davis (2001) 70.

80 Repertorium 170, 'Krs 1' = CIG 4430; LANGLoIs (1861) 194; Bent (1891) 258, Nr. 31; OGIS II 580; MAMA III 102, Nr. 3; PLRE I 982 s.v. 'Fl. Vranius 5'; vgl. RoUgÉ (1966) 296.

81 Zu Matronianus $C T h$ IX 27, $3=C J$ IX 27, 1; PLRE I 568 s.n. 'Matronianus 2'; SCHARF (1990). HILl (1985) schlägt die Identifizierung eines auf einer in Yanihan gefundenen Inschrift erwähnten Matronianus mit dem comes Isauriae vor; dagegen überzeugend HILD / HeLlenkeMPER (1986) 82 ff. Zur I Armeniaca ND or. VII 13. 49. Spätestens nach Kaiser Jovians Vertrag mit Shapur II. von 363 waren die beiden armenischen Grenzlegionen mobilisiert, also als pseucocomitatensische Legionen in die Feldarmee eingegliedert worden, vgl. HOFFMANN (1969) 420-423; ElTON (1996) 95; WoOds (1998) 111; doch zumindest die $I$ Armeniaca war nach Eutychianus frg. 1 (FHG IV 6) bereits zu unter Julian mobilisiert worden, so LENSKI (1999b) 313, Anm. 21. Als eine pseudocomitatensische Legion unterstand sie dem mag. mil. per Orientem, ND or. VII 49. Woods (1998) 110f. vermutet - ohne Quellengrundlage - einen komplexeren Zusammenhang der Mobilisierung der armenischen Legionen, da die I Armeniaca nach der Mauer-Inschrift von Anemurium im Jahre $382 \mathrm{dem}$ comes Isauriae untergeben gewesen sei, somit zu diesem Zeitpunkt noch eine Sprengelarmee gewesen sein müsse. Dies geht aus dieser Inschrift jedoch keineswegs hervor. Da erst zum Abfassungszeitpunkt der ND um 396 die Existenz der pseudocomitatensischen armenischen Legionen gesichert sei, seien sie wohl erst zu einem späteren Zeitpunkt als 363 zusammen mit der I Isaura zu pseudocomitatensischen Legionen erhoben worden, wohl um die Usurpatoren Maximus 388 und Eugenius $394 \mathrm{zu}$ bekämpfen, vgl. JONES (1964) II 609. Sie seien also, so Woods gegen LENSKI l.c., wohl zunächst ohne Beförderung wegen der Unruhen nach Isaurien verlegt worden, und zwar wegen des Aufstands von 375 (s.o.), als limitanei unter dem Kommando des comes Isauriae. Valens habe die 
Mauern der Küstenstadt Anemurium verstärken. Vermutlich wurde die I Armeniaca nach Anemurium beordert, um die Kampfkraft der Isauria II und III nicht einzuschränken. Eine auf der dem Meer zugewandten Seite der Mauer enthält die Bauinschrift. ${ }^{82}$ Die Mauer wurde laut Inschrift in großer Eile errichtet ( $\left.\tau \alpha \chi \chi 1 \sigma \tau \alpha\right)$. Dies könnte man ausschließlich als Eigenlob der Erbauer interpretieren - „wir haben die Mauer wirklich rasch errichtet“. Doch die Tatsache, daß die Inschrift nur grob als Palimpsest auf einem Türsturz-Block eingeritzt ist, läßt auf eine aktuelle Bedrohung der Stadt und die tatsächlich in großer Eile vorgenommene Aufmauerung schließen. ${ }^{83}$ Bei der Inschrift handelt es sich um einen von dem ursprünglichen Platz an der Mauer auf der Seeseite ins Meer herabgestürzten Block, der 1968 kurzfristig freikam. Die Mauer von Anemurium ist eine Generation nach der Verstärkung an der Seeseite im Jahr 425 durchbrochen worden, um den Bau der Apostelkirche zu ermöglichen, was auf eine wieder eingekehrte Ruhe zu dieser Zeit schließen läßt. ${ }^{84}$

militärische Besatzung Isauriens zu verstärken versucht, weshalb sich die Ankunft der beiden armenischen Legionen auf 375 herabdatieren ließe. Auch HellenKEMPER (1986) 630 vermutet eine längere Stationierung der legio I Armeniaca in Anemurium, da eine gute Versorgungsbasis aus dem Umland vorhanden und die Festung Palae in der Nähe war. Damit sei eine eventuelle Zangenbewegung mit den in Seleucia stationierten Legionen nach Isaurien möglich gewesen. Für diese Spekulationen fehlt allerdings die Quellengrundlage, und eine Mobilisierung der armenischen Legionen in den frühen 360er Jahren ist aufgrund des Feldzugs Julians oder des Friedensvertrags Jovians als weitaus wahrscheinlicher anzunehmen. Des weiteren schließt Woons (1998) $110 \mathrm{f}$, aus dem Umstand, daß die pseudocomitatensischen Legionen immer paarweise operierten, ELTON (1996) 91, auf eine Anwesenheit von fün Legionen ab 375 in Isaurien. Doch angesichts der Bedrohungslage durch Goten und Perser sowie der wahrscheinlichen Mobilisierung der I Isaura zur Unterdrückung der Usurpation des Procopius, s.o., ist diese Annahme wenig wahrscheinlich.

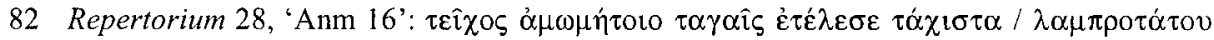

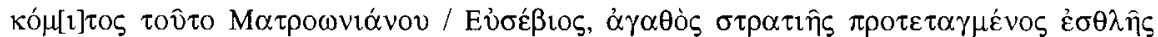

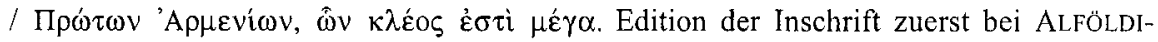
Rosenbaum (1972); der von ihr bemängelte metrisch unsaubere Creticus in der dritten Zeile wird aufgelöst von Jones (1972) und MERKELBACH (1973). Sie schlagen die Version

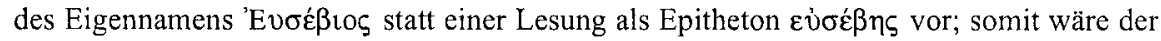
Kommandant der Legion Eusebios der Erbauer im Auftrag des Matronianus. Dies ergibt auch deshalb Sinn, weil im Text vom Kommandeur der Legion die Rede ist, und ein solcher kann Matronianus, dessen Rang als comes in der zweiten Zeile genannt und dessen Amt als comes Isauriae durch CTh IX 27, $3=C J$ IX 27, 1 bezeugt ist, damit nicht gewesen sein. Repertorium 28, 'Anm 16' bringt allerdings noch die alte Lesart, Woods (1998) 110, Anm. 6 folgt der neuen, schließt aber auf einen Status der Armenia I als pseudocomitatensische Legion, s.o.

83 Alföldi-Rosenbaum (1972) 183; Hild / Hellenkemper (1986) 82, Anm. 36.

84 TIB 5,1 190 s.v. 'Anemurion'. 
Matronianus begegnet uns noch in einem Gesetz aus demselben Jahr $382 .{ }^{85} \mathrm{Er}$ bekam darin die Anweisung, dafür zu sorgen, daß der frühere dux Natalis, offenbar sein Vorgänger im Amt, sich in der Provinz Isauria wieder einzufinden habe und Gelder, die an ihn und an seine Untergebenen geflossen sind, zurückzahlen solle. $\mathrm{Ob}$ die Anordnung ausgeführt wurde, erfahren wir nicht. Das Gesetz wirft ein Schlaglicht auf die Korruption, die auch in der nun zur Frontprovinz gewordenen Isaura um sich griff. $^{86}$

\subsubsection{Die Befestigung des Thekla-Heiligtums}

Als die aus dem Westreich stammende Nonne Egeria auf dem Rückweg ihrer Pilgerreise von Jerusalem im Mai 384 über Tarsus auch nach Seleucia gelangte, besuchte sie das dortige Thekla-Heiligtum, um die Akten der Märtyrerin zu lesen. ${ }^{87}$ Es war zu dieser Zeit ein übliches Ziel für Pilgerinnen von und nach Jerusalem. ${ }^{88}$ Durch die Konsolidierungspolitik des Theodosius I. war gerade eine Phase der Ruhe eingekehrt. Die Isaurier blieben dennoch eine ständige Bedrohung: Das Heiligtum war vor Egerias Ankunft mit einer starken Mauer versehen worden. In ihrem Bericht schreibt sie:

„[...] Die Mauer zum Schutz der Kirche wurde angelegt wegen der Isaurier - denn sie sind sehr schlecht und begehen oft Diebstähle -, damit sie nicht versuchen, irgend etwas gegen das zugehörige Kloster zu unternehmen" ${ }^{89}$

85 CTh IX 27, 3 = CJ IX 27, 1; bereits SEECK (1919) 259 hatte das im Manuskript des Codex genannte dux et praeses Sardiniae zu Isauriae emendiert, was nun durch die Inschrift bewiesen ist; ebenso Wilhelm EnssLIN, s.v. 'Matronianus 2', RE XIV (1930) 2309. s.v. 'Natalis 3', RE XVI 2 (1935) 1799; PLRE I 617 s.n. 'Natalis'.

86 Zur Unwirksamkeit der Anti-Korruptionsgesetze vor dem Hintergrund der Kontroverse um den spätantiken ,Zwangsstaat" vgl. DEMANDT (1997).

87 Pereg. Aeth. XXII 2. XXIII 1. Martindale (1980) 474 s.n. 'Aetheria'. Die vierjährige Reise der Egeria läßt sich auf die Jahre 381-384 eingrenzen, vgl. Devos (1967) u. RöWEKAMP Pereg. Aeth. (1995) 21-29: Eckdaten sind die Jahre 363 und 540 n. Chr.: Nisibis ist schon von den Persern beherrscht (Pereg. Aeth. XX 12); damit ist die Übergabe durch Jovians Frieden gemeint. Die Restaurierung der sinaitischen Klöster durch Justinian zwischen 527 und 557 ist noch nicht erwähnt, und Antiochia hat sie noch vor der Zerstörung durch die Perser gesehen. Aufgrund der Nennung der Bischöfe von Batanis und Edessa (Pereg. Aeth. XIX 5. XX 2), der Datierung des Osterfests und der Beschreibung des Verlaufs der Osterzeit wohl des Jahres 383, das sie in Jerusalem verbrachte, ist die enge Datierung möglich.

88 Vgl. Theod. h. r. XXIX 7.

89 Pereg. Aeth. XXIII 4: Sed ut redeam ad rem, monasteria ergo plurima sunt ibi per ipsum collem, et in medio murus ingens, qui includet ecclesiam, in qua est martyrium, quod martyrium satis pulchrum est. Propterea autem murus missus est ad custodiendam ecclesiam propter Hisauros, quia satis mali sunt et frequenter latrunculantur, ne forte 
Die drei großen, für den Pilgerbetrieb angelegten Zisternen befanden sich innerhalb dieser Mauer. Heute sind jedoch nur noch Spuren der Fundamente in einer Entfernung von 40-60 Metern rund um die Thekla-Basilika erhalten. ${ }^{90}$

Das Heiligtum blieb in der Folgezeit durch die isaurischen Banditen bedroht: Die Miracula schildern einen nach der Jahrhundertwende erfolgten Angriff. Sie konnten jedoch von einer Reitereinheit (welche wohl nach dem Abfassungsdatum der Notitia Dignitatum 396 dem comes Isauriae angegliedert worden war) besiegt werden. Der Bischof von Seleucia, Dexianus, ließ als Vorsichtsmaßnahme schließlich in aller Heimlichkeit den Kirchenschatz, auf den es die Räuber abgesehen hatten, in die Stadt bringen - sehr zum Mißfallen der Heiligen, mithin ihres Hagiographen. Es handelte sich dabei wohl um diverse Gold- und Silbermünzen sowie die liturgischen Gerätschaften. ${ }^{91}$ Auch das Küstenstädtchen Selinus wurde trotz guter Verteidigungsanlagen geplündert und blieb bedroht, ebenso Dalisandus im Landesinnern. ${ }^{92}$

\subsubsection{Die Befestigung von Einzelgehöften}

Angesichts der fortwährenden Bedrohung wurden zu dieser Zeit auch Einzelgehöfte befestigt, wie beispielsweise das im Hochland zwischen Olba und Seleucia gelegene Weiler Gökburç. Bei dem Gehöft fällt die geringe Mauerstärke auf; offensichtlich rechnete man nicht damit, daß die potentiellen Belagerer über nennenswerte Maschinerie zum Belagern und Mauerbrechen verfügten. ${ }^{93}$ Auch die seit hellenistischer Zeit in dieser Gegend zahlreich vorhandenen Turmbauten konnten ihren Zweck wieder erfüllen. ${ }^{94}$ Der Wohnturm in Olba war bereits im 3. Jahrhundert neu befestigt worden. ${ }^{95}$

propter Hisauros, quia satis mali sunt et frequenter latrunculantur, ne forte conentur aliquid facere circa monasterium, quod ibi est deputatum.

90 MAMA II 1-89; weitere Beschreibungen des Heiligtums bei DAGRoN Mir. (1978) 55-79; KötTIng ( ${ }^{2} 1980$ ) 145-151; WiLkInSON Pereg. Aeth. (1981) 288-292; Hansgerd HellenKEMPER, RBK IV (1990) 228-235; TIB 5,1 441 ff.; MITCHELL (1995 b) 116; s.o. Kap. II.7.

91 Mir. 28. 32.

92 Mir. 26f. mit Dagron (1978) 112, 359, Anm. 1; TIB 5, 1 407f. s.v. 'Selinus' datieren diesen Vorfall auf die Zeit der Abfassung der Mir. um 450.

93 HeLlenKeMPER (1986) 631f.; HiLd / HELLENKEMPER (1986) 60f.

94 HOPWOOD (1986); DURUGÖNÜL (1998).

95 S.o. Kap. II.3. 


\subsection{Ein innerisaurischer limes?}

Wie bisher gezeigt wurde, deutet eine Vielzahl von Quellen auf eine große Unsicherheit in Isaurien hin. Zuweilen entsteht der Eindruck, daß das Land völlig von Banditen beherrscht wurde und nur die Städte Inseln der Zivilisation in einem Meer von Barbarei gewesen seien - so zeichnet der Autor der Miracula die Situation. ${ }^{96}$ Zwei weitere Quellen geben Anlaß zu der Vorstellung, wonach Isaurien insgesamt eine Art Enklave mitten im römischen Reich gewesen sei, umringt von Militärposten: In der Historia Augusta findet sich die Schilderung, daß Isaurien nach den großen Aufständen durch eine neue Art von Wachen gleichsam wie durch einen limes eingeschlossen wurde, wobei sein Inneres von Orten, nicht von Menschen verteidigt wurde. ${ }^{97} \mathrm{Im}$ Titelblatt der Notitia Dignitatum für den comes Isauriae ist auf der nördlichen Taurusseite eine Reihe von architektonischen Symbolen zu sehen, die auf eine regelrechte Festungskette schließen lassen und mit der so verstandenen Stelle der Historia Augusta konform gingen. Damit spiegle sich, so Hansgerd HELLENKEMPER, die militärische Realität zur Zeit der Abfassung des Werkes wider. Es handle sich dabei wohl um die bei Ammian genannten municipia et castella, wobei es unklar sei, ob es sich um eine Festungskette innerhalb Isauriens oder am Nordrand des Taurus im flachen Lykaonien handelte. ${ }^{98}$ Das von Bassidius Lauricius befreite Kastell mitten in Isaurien bei Antiochia deutet allerdings darauf hin, daß eine starke Durchdringung Isauriens von Festungen bereits im ausgehenden 3. Jahrhundert erfolgt war, denn vor der Wiedereroberung hatte es sich bereits lange ( $\mathrm{diu}$ ) in der Hand von isaurischen Banditen befunden. ${ }^{99}$ In jedem Falle sei Isaurien nach Süden hin durch die in Seleucia oder Umgebung stationierten Legionen abgesichert gewesen, was den Ring geschlossen habe.

Unterzieht man die Stelle der Historia Augusta jedoch einer genaueren Lektüre, so fällt zunächst auf, daß hier von militärischen Limeskastellen im engeren Sinne keine Rede ist. Das locis defensa, non hominibus ist auf regio bezogen. Somit ist die isaurische Bergwelt nicht von den Römern durch eine Festungskette umringt, sondern umgekehrt ist diese vor römischen Einfällen geschützt. Dies legt auch der nachfolgende Kontext nahe. Zudem macht das quasi deutlich, daß limes hier überhaupt in metaphorischem Sinne gebraucht ist. ${ }^{100}$ Die Interperetation des novum genus custodi-

96 Mir. 13. 32 mit DAGRON (1978) 114, 120.

97 SHA trig. tyr. 26, 6: Denique post Trebellianum pro barbaris habentur; etenim in medio Romani nominis solo regio eorum novo genere custodiarum quasi limes includitur, locis defensa, non hominibus.

98 ND or. XXIX, zur Beschreibung s.o. Kap. III.1.10; HeLLENKEMPER (1986) 629 mit Verweis auf Amm. XIV 2, 5. 10.

99 ILS 740; s.o. III.1.10. u. IV.2.2.

100 Hierauf verweisen u. a. RougÉ (1966) 207 ff., SCHWARTZ (1970) 234 u. HopwoOd (1986) 344. Diese Interpretation ist plausibel angesichts des folgenden Satzes SHA trig. tyr. 26, 
arum muß also wie folgt lauten: üblicherweise ist ein limes, wie ihn die Römer kennen, durch Menschen (die sich durchaus in befestigten Lagern aufhalten können) gebildet. Im Unterschied hierzu verteidigen sich die Isaurier gegen die Römer durch ihren natürlichen Grenzwall, das Taurusgebirge. Dieses stellt in der Vorstellung des Autors der Historia Augusta eine neue, den Römern bisher nicht untergekommene Art von ,Wachen" dar. ${ }^{101}$

Daß Isaurien als unregierbarer Bezirk gleichsam von einer inneren Front umstellt und damit vom restlichen Reich isoliert war, ist somit zwar ein nicht zuletzt von der Historia Augusta gezeichnetes plakatives Bild, doch es wird selbst in Zeiten größter Unruhen nicht der Wirklichkeit gerecht. Zwar kann es für die zweite Hälfte des 4. Jahrhunderts als zutreffend angesehen werden, daß Isaurien ein regelrechter „Binnenmilitärbezirk“" wurde, in dem ,Militäreinheiten gegen den inneren Feind geführt“ wurden, doch eine Interpretation der militärpolitischen Situation als ,auswegloser Guerillakrieg“, wonach die ,seit Jahrhunderten weitgehend erfolglosen Pazifizierungsversuche fehlgeschlagen" sein sollen, ${ }^{102}$ ist selbst für diese Zeit der extrem groBen isaurischen Aufstände überzeichnet. Die römischen ,Legionen im Bandenkrieg“ konnten zwar den Guerillakrieg im unwegsamen Gelände kaum gewinnen, wie es Ammian schildert, sondern nur in der Ebene größere Erfolge erzielen. Dennoch gibt es, wie die zahlreichen angeführten Belege aufgewiesen haben, auch genügend Gegenbeispiele für eine Übernahme der Guerillataktik durch kleinere Einheiten, wie die

7, worin die Kriegsuntüchtigkeit der Isaurier herausgestellt wird: nam sunt non statura adecori, non virtute graves, non instructi armis, non consiliis prudentes, sed hoc solo securi, quod in editis positi adiri nequeunt.

101 HoHL SHA (1985) 146 übersetzt trig. tyr. 26, 6 mit „es wird nämlich ihr Gebiet, obwohl es mitten im römischen Reich liegt, durch eine neue Art von Wachen wie ein Grenzwehrdistrikt eingeschlossen; wird es doch durch das Gelände, nicht durch Menschen verteidigt" und bezieht damit auch das novum genus custodiarum auf die isaurische regio, dito David MAGIE (1956) 129 in der Loeb-Übersetzung. Hingegen bezieht DE JONGE Amm. (1935) 116 Amm. XIV 2, 13 militibus omne latus Isauriae defendentibus auf l.c. der SHA und damit offenbar auf römische Legionäre. SCHWARTZ (1970) 234 interpretiert diesen limes als die Vorstellung von einer rein defensive Grenze, die in der römischen Grenzpolitik keinerlei Rückhalt finde, sondern mittelalterliche, personenverbandsmäßige Verhältnisse antizipiere, vgl. HoHL SHA (1985) 365. Nach LEWIN (1991) 180 ist der limesBegriff auch auf die Rolle der räuberischen Region insgesamt als „,blocco della circulazione interregionale“ bezogen. Für Rougé (1966) 285 u. MARAsCo (1983) 218 ist die Formulierung ein weiterer Beweis ihrer These einer Rückprojektion der SHA von Verhältnissen des späten 4. Jhs. auf das 3., da die Stadtbefestigungen erst zu dieser Zeit inschriftlich bezeugt seien. Doch die befestigten Lager haben einen rein militärischen, offensiven Charakter und sollten nicht mit den defensiven Stadtbefestigungen gleichgesetzt werden. Zur Diskussion der Rückprojektions-These s.o. Kap. IV.1.4. 
erfolgreiche Bekämpfung der isaurischen Marodeure durch Aushungern und Besetzung von strategischen Positionen wie dem Fort in Antiochia im isaurischen Bergland.

Die Banditen wurden nach den militärischen Auseinandersetzungen im 4. Jahrhundert zwar nicht weiter verfolgt, doch das Militär behielt im großen und ganzen die Kontrolle über die Situation. Die Städte blieben unbehelligt, denn angesichts ihrer Mauern waren sie für die Isaurier mangels schwerem Gerät nicht zu überwinden. Taktisch durch Guerillatechnik überlegen, waren die Isaurier strategisch den römischen Truppen unterlegen. Dies zeigt auch der (unten geschilderte) erfolgreiche und rasche Einsatz des comes rei militaris oder comes Isauriae Arbazacius ${ }^{103}$ gegen die Isaurier, der ihre Dörfer niederzubrennen begann. Insofern konnten sich die Isaurier letztendlich nicht gegen die Legionen durchsetzen. Sie ließen sich statt dessen auf Friedensverhandlungen ein und stellten Geiseln. Dieser Umstand, daß nur die Isaurier, nicht aber die Römer Geiseln stellten, verdeutlicht, daß es sich in Isaurien nicht um eine freie, autonome Zone gehandelt haben kann.

Exemplarisch für die unsichere Lage in Isaurien zu dieser Zeit und zugleich ein Beweis für die Notwendigkeit, die isaurische Gesellschaftsstruktur differenzierter als lediglich in der Kategorie des Widerstands gegen Rom zu betrachten, ist das Schicksal des Isauriers Dionysius. Dieser war ein Schüler des Libanius. Der berühmte Rhetor verfaßte eine Lobrede auf den Vorgesetzten des Dionysius, den comes Isauriae Palladius. ${ }^{104}$ Dionysius lebte, bevor er zu Libanius nach Antiochia kam, mit seiner Familie in Isaurien. Im Jahr 355 ermordeten Banditen seinen Vater, ${ }^{105}$ wohl im Zuge des Aufstands von 354. Die Mutter des Dionysius und ihr zweiter Ehemann hatten sich darauf hin des väterlichen Vermögens bemächtigt, Dionysius war leer ausgegangen und gelangte nach Antiochia zu Libanius, der ihn als Schüler aufnahm. Kurz darauf hoffte er mit Hilfe des comes Isauriae, zu dieser Zeit Castricius, einen Teil seines Vermögens zurück zu erhalten, offenbar erfolglos, da er noch 357 die Unterstützung des Libanius in dieser Sache benötigte. Im Jahr 363 wurde er selbst Rechtsanwalt und konnte in einem glücklichen Prozeß wieder an seine Güter heran. ${ }^{106}$ Eine daraufhin, im Jahr 364 angebotene Tätigkeit in Castricius' officium lehnte er ab, um sich, wie er es ausdrückte, ganz seinen Landgütern widmen zu können. Der wahre Grund für die Verweigerung dürfte jedoch eine Verurteilung wegen Frauenraubs

103 Comes Isauriae nach Woods (1998) 114; nach PLRE II 127f. s.n. 'Arbazacius 1' eventuell comes rei militaris.

104 PLRE I 258 s.n. 'Dionysius 6'; SEECK (1906) 121f.

105 Lib. ep. 319, 426, 1169.

106 Lib. ep. $1168,1438$. 
gewesen sein, welcher er sich durch die Fürsprache des Libanius entziehen konnte. ${ }^{107}$ Im folgenden Jahr 365 war er rehabilitiert und wieder als erfolgreicher Anwalt tätig. ${ }^{108}$ Sein Lebenslauf macht deutlich, daß selbst in der Zeit der großen Aufstände ein (sogar gerichtlich beklagter) Isaurier Karriere machen konnte, und von einer Dichotomie zwischen isaurischer und römischer Gesellschaft insgesamt nicht die Rede sein kann. Selbst in den Miracula, einer mit Übertreibungen nicht geizenden Hagiographie, werden die urbanisierten Isaurier zuweilen auch als Opfer ihrer räuberischen Landsleute geschildert, und Angehörige isaurischer Geiseln waren angesehene, integrierte Polisbürger. ${ }^{109}$

Mit den unter Theodosius vorangetriebenen Befestigungen und der Politik, isaurische Einheiten im Militär zu bilden (s.o.), wurde eine vorübergehende Beruhigung der Situation zwischen 382, der Befestigung von Anemurium, und 396, dem Wiederaufflammen der isaurischen Raubzüge, erreicht. ${ }^{110}$ Doch nach der Regierungszeit des Theodosius setzten sich die isaurischen Plünderungen mit unverminderter Härte fort und erreichten zuvor nicht gekannte geographische Ausmaße: der gesamte östliche Mittelmeerraum wurde unsicher gemacht. Eine Erklärung hierfür findet sich in der sogenannten Gainaskrise, auf die nun näher eingegangen werden soll.

\subsection{Die Gainaskrise und die Isaurierunruhen}

Wie bereits die Schwächung des römischen Militärs durch isaurische Aufstände am Vorabend der Schlacht von Adrianopel, so steht umgekehrt der Aufstand des Goten Tribigild in Kleinasien und sein Kampf gegen den zunächst romtreuen Goten Gainas $^{111}$ in engem Zusammenhang mit den Isaurierunruhen der Jahre 396-408: Ihre

107 Lib. ep. 1168, 1169, 1204, 1237, 1238, 1249; Frauenraub: CTh VIII 24, 2, ein Vergehen, das später unter Todesstrafe stand: $C J$ IX 13.

108 Lib. ep. 1470, 1501.

109 Mir. 13, Z. 12 ff.

110 Nach Woods (1998) 112 sind von 375-396 keine Unruhen in Isaurien vermeldet, doch dagegen spricht die hastige Verstärkung der Mauer von Anemurium, s.o.

111 Zos. V 13-22; Eun. frg. 66-69; Claud. in Eutr. II; Joh. Ant. frg. 190; SEECK (1921) V 359f; Stein (1928) 306 ff. 315. 318; Demandt (1989) 158f.; Tiersch (2002) 265-308; Synes. Prov. mit Clauss (1980) 133 ff.; Cameron / Long (1993) 225. 245; andere Identifizierung bei Wolfgang HAGL, Arcadius Apis Imperator, Synesios von Kyrene und sein Beitrag zum Herrscherideal der Spätantike (= Frankfurter althistorische Beiträge 1), Stuttgart 1997, 56, wonach Synes. de regno gegen Gainas gerichtet sei, ablehnend hierzu die Rezensionen von Andreas Goltz, Gymnasium 107 (2000) 26I-264 u. Noel LENSKI, BMClR 3 (1998): „While his work will be read with interest by devotees of Synesius, it will not pose a serious challenge to what could now be called the new orthodoxy". Synesius attackiert in seiner Kaiserkritik de regno die "Skythen" in der römischen Armee. De 
Angriffe auf Pamphylien wurden durch die Schwächung der römischen Truppen und das durch Tribigilds Aufstand hervorgerufene Chaos erleichtert. ${ }^{12}$ Tribigild kommandierte (wohl im Rang eines comes rei militaris mit Sitz im phrygischen Nacoleia) gotische Föderaltruppen, die dort 386 unter Theodosius I. angesiedelt worden waren. Im Frühjahr 399 entschlossen diese sich, unter seiner Führung in das fruchtbarere Pisidien einzufallen, da sie für die Teilnahme an einem vorangegangenen Hunnenfeldzug von dem mächtigen Eunuchen im Amt des praepositus sacri cubiculi Eutropius eine Sonderzahlung verlangt hatten, die sie nicht bekommen hatten. ${ }^{13}$ Dieses Ereignis führte letztlich zum Sturz der herrschenden Gruppe in Konstantinopel unter Eutropius. Der zu seiner Bekämpfung von Eutropius eingesetzte magister militum Gainas nutzte die Gunst der Stunde, um sich selbst zu einer machtvollen Position

providentia dagegen ist eine Allegorie auf den Gainas-Aufstand, vgl. DEMANDT (1989) 157. Nach HEATHER (1988) hat Synesios mit seiner ,anti-skythischen Tirade“ nicht die Goten unter Fravitta und Gainas gemeint, sondern seine Kritik habe vielmehr auf die mit Alarich nach Westen ziehenden Goten abgezielt. - Zu Gainas u. Tribigild vgl. PLRE I 379f. s.n. 'Gainas'; ergänzend Martindale (1980) 485; PLRE II 1125 s.n. 'Tribigild'; Seeck (1921) V 306 ff.; Stein (1928) 359 ff.; Schmidt (1941) 433 ff.; Demougeot (1951) 223 ff.; Demandt (1970) 732 ff; Frank M. Clover, 'Count Gainas and Count Sebastian' in: $A J A H 4$ (1979) 65 ff.; AlBerT (1984) 87-149 mit Replik von CESA (1994) 76-90; LiebESCHUETZ (1989). GLUSCHANIN (1989) nimmt eine Zwischenposition in der Kontroverse um den von AlBERT (1984) passim u. 180 verneinten „Antigermanismus“ und die Idee eines Kampfes zwischen einer germanenfreundlichen und einer germanenfeindlichen Partei am Hof ein: Während es statistisch keinen überhöhten Anteil von Germanen im Heer gegeben habe, sei der Antigermanismus bereits in der Politik des Theodosius I. greifbar. Resümierend WOLFram $\left({ }^{3} 1990\right) 175$ ff. Gegen die v.a. von ALBERT vertretende Skepsis am Antigermanismus und hinsichtlich der Isaurier von ELTON $(2000 \mathrm{a} / \mathrm{b})$ übernommen - nicht die Ethnizität, sondern die Nähe zur Macht sei Motiv und Konstitutive gewesen, wendet sich diese Arbeit, s.u. Kap. IV.2.

112 Zos. V 25, 2: 'E

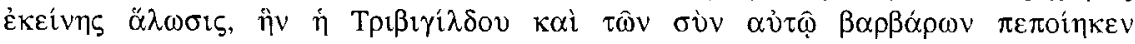
$\dot{\varepsilon} \pi \alpha v \alpha \dot{\sigma} \sigma \alpha \sigma \iota \varsigma$. Vgl. auch LENSKI (1999a) 425.

113 Claud. in Eutr. II 236f. 274 ff.; Philostorg. XI 8; Eun. frg. 67, 10f.; Soc. h.e. VI 6; Soz. h.e. VIII 4; Zos. V 13 ff;; Joh. Ant. fr.g. 190; Wilhelm ENSSLIN, s.v. 'Tribigild', RE VI A2 (1937), 2403 ff.; PLRE II 1125f. s.n. 'Tribigildus'; PLRE II 440-444 s.n. 'Eutropius 1'; ALBert (1984) $89 \mathrm{ff}$; zu Eutropius und der politischen Funktion der Hofeunuchen vgl. Helga Scholten, Der Eumuch in Kaisernähe. Zur politischen und sozialen Bedeutung des praepositus sacri cubiculi im 4. und 5. Jh. n. Chr., Frankfurt a.M. 1995; Dirk SCHLINKERT, Ordo senatoris und nobilitas. Die Konstitution des Senatsadels in der Spätantike (= Hermes Einzelschriften 72), Stuttgart 1996, 254 ff. mit Anm. 53. 266-270 zu Eutrop. Beide Arbeiten betonen gegen die v.a. bei Claud. Eutr. und Zos. V 12, 1 formulierte scharfe Kritik die Funktion des PSC als stabilisierenden Faktor im kaiserlichen Herrschaftssystem gegenüber den zentrifugalen Kräften der mächtiger werdenden Heermeister. 
aufzuschwingen und zu versuchen, wie Stilicho im Westen, Kontrolle über den östlichen Teil des Imperiums zu erreichen. Insofern bildet der Gainasaufstand eine „Nahtstelle" in der Geschichte des östlichen Heermeisteramts. ${ }^{114}$

Gainas war ein terwingischer Gote ${ }^{115}$ von niederer Abkunft und Arianer. Im Auftrag Stilichos hatte er nach dem Sieg über den Usurpator Eugenius als comes rei militaris dem Arcadius nach Konstantinopel Truppen zugeführt. Sein Zug in die Hauptstadt zielte nach dem mittlerweile bezweifelten Urteil des Zosimus jedoch eher auf die von Stilicho in Auftrag gegebene Ermordung des praefectus praetorio Rufinus. ${ }^{116}$ Im Jahr 399 zum magister utriusque militiae erhoben, schickte ihn Eutropius nach Phrygien, um Tribigilds dortigem Treiben ein Ende zu bereiten. Stattdessen vereinigte er sich aber mit Tribigild und beseitigte schließlich Eutropius selbst. ${ }^{117}$ Zuvor war es Eutropius noch gelungen, den Goten ihre Privilegien zu entziehen und ihre unabhängige Machtstellung zu neutralisieren. Dies gab dem westgotischen Heerführer Alarich Anlaß, Griechenland zu plündern und sich schließlich nach Westen zu wenden. Auch Tribigild lehnte die von Eutropius angebotenen Stillhaltegelder ab und setzte seinen Aufstand fort. Daß der gesamte Osten an die Goten fiel, wurde laut Zosimus dadurch verhindert, daß Tribigild es zunächst vorzog, auf eigene Faust zu plündern. Der Grund hierfür dürften einmal mehr Versorgungsschwierigkeiten seines Volkes gewesen sein,

114 DEMANDT (1970) 733.

115 Als vermutlicher comes rei militaris war Tribigild Gainas unterstellt und, als dessen Gefolgsmann und Verwandter, Soc. h.e. VI 6, 5, Soz. h.e. VIII 4, 2, wohl ebenfalls ein Terwinge, so LiebeSCHUETZ (1986) 467 gegen AlBERT (1984) 89, der ihn nach Claud. in Eutr. II 153f. 158f. 196f. als Greuthungen ansieht.

116 Zos. V 7; PLRE I 379 s.n. 'Gainas'. 778-781 s.n. 'Rufinus 18'; DEMANDT (1989) $158 \mathrm{f}$. Die neuere Forschung bestreitet eine Beteiligung Stilichos an den Vorgängen im Ostreich in diesen Jahren, vgl. CAMERON (1970) 141 ff. Albert (1984) passim; REBENICH Zos. (1990) 367 , Anm. 36 zu Zos. V 17. Zwar hatte Stilicho 395 gehofft, mit Gainas' Hilfe seinen militärischen und politischen Einfluß auf das Ostreich ausbauen zu können, doch entfremdete sich Gainas in der Folgezeit immer mehr von Stilicho und konsolidierte seine Machtposition im Osten. Es besteht folglich kein Grund, an Zosimos' späterer Aussage zu zweifeln, Gainas habe eigennützig Eutrops Sturz betrieben. Soz. h.e. VIII 7, 3 und Philostorg. h.e. XI 6 führen aus, die Kaiserin Eudoxia habe durch eine dem Arcadius vorgetragene Beschwerde über Beleidigungen des Eunuchen dessen Absetzung ausgelöst; vgl. HoLUM (1982) $62 \mathrm{f}$.

117 Zos. V 13, 1f.; 17, 4. Zos. V 14 unterstellt Gainas, bereits von Beginn seiner Erhebung an mit Tribigild zusammengearbeitet zu haben, gefolgt von PLRE II 1125f. s.n. 'Tribigild': „he acted in collusion with Gainas". Dies wird zum Teil bezweifelt. ALBERT (1984) 113 ff. vermutet ein Zusammengehen der beiden erst nach der Niederlage des anderen Heermeisters Leo; ablehnend PASChOud Zos. (1986) III1 125f. Nach CeSA (1994) 85 unter Verweis auf Eun. frg. 67, 10 haben Gainas und Tribigild sogar mit Fravitta zusammengearbeitet, dem mag. mil. per Orientem, der sich zu diesem Zeitpunkt im Kampf gegen die Isaurier befand und später Gainas besiegte, s.u. 
denen er nachzugeben hatte, wenn er seine Autorität als Heerführer behalten wollte. Doch eine Niederlage bei der pamphylischen Stadt Selge, ${ }^{118}$ die ihm zwei lokale Autoritäten, Valentinus und Florentius in einem Engpaß beigebracht hatten, ${ }^{119}$ stoppte den Plünderungszug. Tribigild konnte sich zunächst von den Einwohnern Selges, denen offenbar mehr am eigenen Profit als dem Allgemeinwohl gelegen war, die Freiheit erkaufen. Nach weiteren Plünderungen wurde er nochmals von diesen an der Grenze zu Isaurien zwischen den Flüssen Melas und Eurymedon eingeschlossen. Tribigild schickte nun an Gainas um Hilfe, der seine eigenen barbarischen Truppen den Legionären des anderen Heermeisters Leo, welche die Pamphylier entsetzen sollten, nachschickte und die Streitmacht des Leo aufrieb. Tribigild konnte abermals entkommen und die phrygischen Städte weiter bedrohen. ${ }^{120}$

Die aufrührerischen Goten Gainas und Tribigild rückten gegen die Hauptstadt vor und zwangen den Kaiser zu Verhandlungen: In einer Kirche bei Chalkedon oder in der Hagia Sophia in Konstantinopel ${ }^{121}$ mußte Arcadius die Anführer der germanenfeindlichen Hofpartei ${ }^{122}$ (den praefectus praetorio und Consul Aurelianus, Saturninus, und den comes sacrarum largitionum Johannes) ausliefern und dem comes Gainas selbst eine Position als oberster Heerführer einräumen. Zudem forderte Gainas von Arcadius die Beseitigung des Eutropius. Seine Designation zum Consul für 399 und die Verleihung des Patricius-Titels an den Eunuchen durch den Kaiser war bereits dem auf Wahrung seines Einflusses bedachten Hofadel zu weit gegangen. Er wurde darauf zunächst nach Cypern gebracht, was sein Leben jedoch nicht rettete; er wurde

118 Zos. V 14f. mit Paschoud Zos. (1986) III1188 ff.; Claud. Eutr. II 153 ff.; Zu Selge vgl. MAGIE (1950) I 264f. II 1137, Anm. 13; Strab. XII: Selge war noch unzugänglicher als Termessos, hoch über dem Westufer des Eurymedon, nur über einen langen und schmalen Weg erreichen, war deshalb im Verkehr nach Norden orientiert; HALL (1972) 216.

119 Zos. V 15f.; Wilhelm ENSSLIN, s.v. 'Valentinus 15', RE VII A 2 (1948), 2275f.; PLRE II 1139 s.n. 'Valentinus 1'; Otto SEECK, s.v. 'Florentius 11', RE VI, 2 (1909), 2758; PLRE II 477 s.n. 'Florentius 2'.

120 Zos. V 17, 1; Eun. frg. 67, 5 ff. Nach Paschoud Zos. (1986) IIIl 133 u. ReBENICH Zos. (1990) 367, Anm. 32 sei dies eine andekdotenhafte Erzählung, die an die LydiusGeschichte in Cremna erinnere. Doch dieser Umstand muß nicht automatisch deren Historizität in Frage stellen; vgl. auch Hopwood (1989) 196.

121 Paschoud Zos. (1986) IIII 142; Rebenich Zos. (1990) 368.

122 Das Konzept zweier sich bekämpfender Parteiungen ist in jüngerer Zeit mehrfach in Frage gestellt worden, vgl. ALBERT (1984) 180. Dieser Zweifel wird im Hinblick auf die Isaurier in Konstantinopel nun auch von ELTON $(2000 \mathrm{a} / \mathrm{b})$ geäußert. Jedoch betont die Mehrzahl der Quellen immer wieder ausdrücklich die Ethnizität der Goten und Isaurier, und ein Ergebnis dieser Arbeit ist, daß die ethnische Herkunft im Falle der Isaurier doch eine wesentliche Rolle bei deren gescheiterter Integration spielte, s.u. Kap. IV.2.2. 
auf Betreiben des Gainas hingerichtet. ${ }^{123}$ Im Jahr 400 besetzte Gainas Konstantinopel nun doch gegen den Widerstand der Bevölkerung, die Milizen bildete. Als der arianische Gote auch noch den Klerus gegen sich aufbrachte, indem er gegen den Willen des Johannes Chrysostomos (der seine Ernennung Eutropius verdankte) eine katholische Kirche für seine Arianer verlangte, kam es am 12. Juli 400 zum Massaker an seinen 7000 Soldaten. ${ }^{124}$ Tribigild war zuvor gestorben. ${ }^{125}$ Mit dem Rest seiner Truppe floh Gainas nach Thrakien, versuchte dann aber, mit Schiffen über den Hellespont nach Kleinasien zurückzukehren.

Gegen ihn wurde wiederum ein terwingischer Gote, der magister militum per Orientem Fravitta eingesetzt. ${ }^{126}$ Er war mit einer Römerin verheiratet und hatte, wiewohl Heide, seine Treue gegenüber dem Reich bereits 391 am Hof des Theodosius I. unter Beweis gestellt, als er den aufständischen Goten Eriulf getötet hatte. ${ }^{127}$ In den

123 Zos. V 17f.; Claud. 19, 10. 52; 20, 20f.; Philostorg. h.e. XI 6; Soc. h.e. VI 5; Soz. h.e. VIII 7, 2 ff; Joh. Ant. frg. 189; CTh IX 40, 17; ALBERT (1984) 43f. 151 ff. mit weiterer Literatur; DEMANDT (1970) 733 ff. (1989) 159. Michael Dewar, 'The Fall of Eutropius' in: $C Q 40$ (1990), 582 ff. weist auf eine Parallele zu Juvenal 10, 69-72 bei der Schilderung in Claudians Vorwort zum 2. Buch In Eutropium hin; SCHLINKERT (1996) 269f. sieht Gainas an der Spitze der Adelsfraktion am Hof, die den ,allmächtigen Außenseiter“ Eutrop zu Fall bringen wollte, wogegen allerdings die Auslieferung der Gruppe um Aurelianus spricht.

124 Theod. lect. h.e. V 32; abweichende Zahlen in anderen Quellen. Nach LiEBESCHUETZ (1989) 281 reflektiert das auf Abzeichnungen aus dem 16. Jh. erhaltene Bildprogramm auf der Arcadiussäule in Konstantinopel den Abzug der geschlagenen Gainas-Goten nach dem Massaker und deutete dies in christlichem Sinne um, wobei das Massaker selbst unterschlagen wird. Die Lehre, die das Ostreich aus der Gainasaffäre gezogen habe, sei gewesen, nicht mehr eine traditionell große Armee zu unterhalten, wenn diese das Risiko der Insurrektion barg. Man zog es fortan vor, den Barbaren Stillhaltegelder zu bezahlen. So sei, wenn auch weniger ruhmreich, das Bestehen des Ostreichs gewahrt worden.

125 Philostorg. h.e. XI 8.

126 Eun. frg. 69, 2; Zos. V 20f.; PLRE I 372f. s.n. 'Flavius Fravitta'.

127 Eun. frg. 59; Zos. IV 56, 1 ff. Nach Woods (1998) 114 ff. gegen Ridley (1970) 93, Nr. 20 u. Anm. 10. Nach Cameron/Long (1993) 224 u. Burns (1994) 173 tat er dies in der Funktion eines comes Isauriae: es sei möglich, daß Fravitta seinen Erfolg gegen die Isaurier errang, bevor er zum mag. mil. per Orientem ernannt wurde. Dies sei beim Kompilieren der Suda verlorengegangen. Doch dieser Konstruktion ist nur mit Mühe zu folgen, sie setzt eine zweimalige Zurückstufung der armenischen Legionen zu limitanei voraus. Zudem steht die eindeutige Aussage von Eun. frg. 69, 2 (= Suda $\Phi$ 681) gegen diese Spekulation: Fravitta wird dort immerhin $\sigma \tau \rho \alpha \tau \eta \gamma o \dot{\zeta} \tau \hat{\eta} \varsigma$ 'A $\alpha \tau 0 \lambda \hat{\eta} \varsigma$ genannt, was trotz der notorischen terminologischen Unschärfe Eunaps nicht auf eine comitativa Isauriae bezogen werden kann. Seine Truppen gegen Gainas, so Woods gegen den Wortlaut der Quelle (wie er selbst konzediert), habe Fravitta direkt aus Isaurien mitgebracht, er sei dann erst für dieses Kommando zum mag. mil. per Orientem ernannt worden. Für rasche Aushe- 
folgenden Jahren hatte er sich Verdienste bei der Bekämpfung isaurischer Banditen in Pamphylien erworben, welche die Provinz bis hinauf nach Karien heimsuchten. Er war in seinen Maßnahmen gegen die Banditen derart erfolgreich, daß der heidnische Autor Eunap ihn zu einem Helden stilisierte und sich zu der Übertreibung veranlaßt sah, das Wort „Räuberei“ sei nach dem Auftritt des Fravitta fast von den Lippen der Leute verschwunden. Zosimus wiederholt das Lob seiner Quelle: Fravitta habe sich in vielen Feldzügen ausgezeichnet und den ganzen Osten von Kilikien bis Phönikien und Palästina von der Heimsuchung durch Räuber befreit. ${ }^{128}$

Fravitta besiegte Gainas im Herbst 400 in einer Schlacht mit seiner Flotte in der Meerenge vor der Chersones und verhinderte damit dessen geplanten Übergang über den Hellespont. ${ }^{129}$ Die Liburnen (kleine Kriegsschiffe), die Fravitta gegen Gainas einsetzte, müssen wegen der kurzen Frist bis zu ihrem Einsatz bereits vorhanden gewesen sein und bildeten nach Auskunft des Zosimus keine offizielle, sondern seine Privatflotte, ,die für eine Seeschlacht ausreichten“. ${ }^{130}$ Gainas zog sich nach der Niederlage gegen Fravitta über die Donau zurück. Dort kam er in einem Kampf mit den Hunnen unter Uldin um.

bungen sei keine Zeit gewesen, und Seleucia war näher an Konstantinopel als Antiochia, der Amtssitz des mag. mil. per Orientem.

128 Eun. frg. 69, 2. 71, 4; Zos. V 20.

129 Eun. frg. 69, 4; Zos. V 20f.; Soz. VIII 4, 19-21.

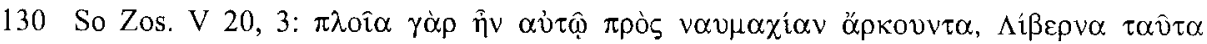
$\kappa \alpha \lambda$ ov $\mu \varepsilon v \alpha$ und fügt einen kleinen Exkurs über diese Liburnen an. Hierzu vgl. REBENICH Zos. (1990) 369, Anm. 45, mit Verweis auf Eun. frg. 81. Die Liburne war eine Bireme, also ein schnelles, mit zwei Riemenreihen ausgestattetes Schiff. Offenbar gebraucht Zos. in seiner archaisierenden Schreibweise diesen Ausdruck nicht mehr als Synonym für „Kriegsschiff“" allgemein, wie es seit Caesar der Fall war (auch wenn er darauf hinweist, daß Trieren seit hellenistischer Zeit nicht mehr üblich waren). Nach Suda $\Lambda 490$ war sie nach Art der Triakontoren mit 30 Ruderern ausgestattet, die Skepsis von Rebenich l.c. und Robert Grosse, s.v. 'liburna', RE XIII, 1 (1926), 143 ff. 144, daß Zos. eine Verwechslung mit diesen unterlaufen sei, ist deshalb nicht angebracht. Zum Begriff vgl. auch Silvio Panciera, 'Liburna' in: Epigraphica 18 (1956) 130 ff; Chester G. STARr, The Roman Imperial Navy 31 B.C.-A.D. 324, Westport, Conn. 1941, 7f. 54. 108; Dietmar KIENAST, Untersuchungen zu den Kriegsflotten der römischen Kaiserzeit, Bonn 1966, 139; Skizzen bei Hans D. C. VIERECK, Die römische Flotte, Herford 1975, 34 ff.; Lionel CAsson, Ships and seamanship in the ancient World, Baltimore ${ }^{3} 1995,141 \mathrm{ff}$. Aufgrund

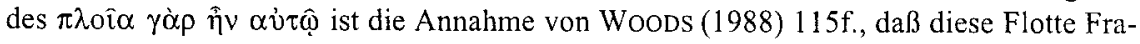
vitta regulär in seiner Eigenschaft als ,,comes Isauriae " unterstanden habe, unwahrscheinlich. Abgesehen davon, daß Fravitta dieses Amt eher nicht innehatte (s.o.), ist weder in der kurz zuvor abgefaßten ND die Rede von einer solchen Flotte, noch kommt sie in sonst einer Quelle explizit vor. Dies muß gleichwohl nicht ausschließen, daß Seleucia über einige Schiffe zur Sicherung der Küsten- und Flußschiffahrt verfügte. 
Nach der Erhebung des Gainas brach der Osten mit der gotenfreundlichen Politik: Zwar wurde Fravitta für den Sieg gegen Gainas im folgenden Jahr 401 mit dem Consulat belohnt. ${ }^{131}$ Unvorsichtigerweise machte er sich dann jedoch die Kaiserin Eudoxia zur Feindin, indem er ihren Günstling Johannes anklagte, zwischen Arcadius und Honorius Unfrieden stiften $z \mathfrak{u}$ wollen. Die Rache des Hofes ließ nicht lange auf sich warten: Um 403/404 wurde er seinerseits auf Betreiben des Höflings Hierax angeklagt, er habe Gainas nach dem Seesieg nach Thrakien entkommen lassen, und wurde im selben Jahr hingerichtet. ${ }^{132}$ Hierax, der nach dieser Intrige praeses in Pamphylien geworden war, soll dann jedoch Eunap zufolge Pamphylien noch mehr als der zuvor stattgefundene Isaurienkrieg des Fravitta geschadet haben. Er wurde schließlich von dem vicarius Asiae Lucius Herennianus wegen exzessiver Bereicherung mit einer Strafe von 4000 Solidi belegt und in der Folgezeit, um 404, nach Ende der IsaurierUnruhen, exekutiert. ${ }^{133}$

\subsection{Die Ausweitung der isaurischen Raubzüge bis nach Palästina}

Da die abtrünnigen Gotenführer im gleichen Zeitraum die pamphylische Ebene durch ihre Machtkämpfe ausgeplündert hatten, wandten sich die isaurischen Banditen nicht mehr nur nach Westen in Richtung Pamphylien bis hin nach Karien, sondern nach Osten bis hinauf in die Armenia Secunda und nach Palästina. ${ }^{134}$ Hierbei zeigte sich einmal mehr die fatale Verquickung mit den Auswirkungen der gleichzeitig marodierenden Goten auf die Situation im Ostreich: Die erste Plünderungswelle im Jahr 396 im östlichen Mittelmeer fiel dabei zusammen mit der Plünderung Griechenlands durch Alarich, und auf den Zusammenhang der Isaurierunruhen nach der Jahrhundertwende mit dem Tribigild-Aufstand weist Zosimus explizit hin. ${ }^{135}$ Der Zeitgenosse

131 Eun. frg. $69,4$.

132 Zos. V 21, 5; Eun. frg. 71; PLRE II 556 s.n. 'Hierax 1'.

133 Eun. frg. 71, 4; PLRE II 546 s.n. 'Herennianus'. Nach BUCK (1998) 44 ist die darauf folgende Exekution des Hierax für seine Attacke auf Fravitta von Eun. wohl unzulässig um 10 Jahre vorgezogen worden, um sie mit dem als korrupt gezeichneten Regime der Pulcheria in Verbindung zu bringen.

134 Pallad. Dial. 11; Joh. Chrysost. ep. ad Olympiadem VI 1. IX 2. 3. 4. XVI 1. XVII 1; ep. 52. 57. 61. 68f. 72. 108f. 114. 120f. 127. 131. 135f. 142. 146 ( $P G 52)$; Hier. ep. 114, 1;

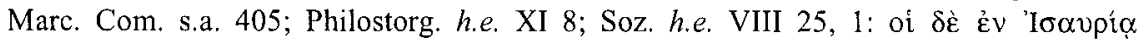

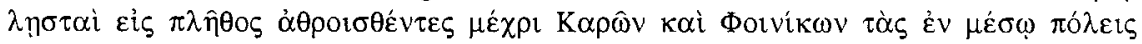

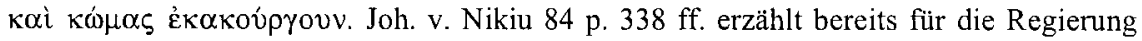
des Theodosius I. von Plünderungen bis nach Palästina, doch hier handelt es sich um eine Verwechslung mit den hier beschriebenen Ereignissen, vgl. MUTAFIAN (1988) 225.

135 Zos. V 25, 2. Zu Alarich vgl. Seeck (1921) V 280f.; Demougeot (1951) 343f.; Rougé (1966) 297 ff.; DemandT (1989) 140f. 
Hieronymus schrieb im Jahr 400 in einem Brief an den Bischof Theophilus von Alexandria, daß die Isaurier Phönizien und Galiläa plünderten und Palästina, selbst Jerusalem bedrohten, und erwähnt den Zusammenbruch der Nahrungsmittelversorgung. ${ }^{136}$

Auf die Isaurier wurde nun nach dem in dieser Angelegenheit letztlich glücklosen Fravitta der Armenier Arbazacius angesetzt. ${ }^{137}$ Er war wegen seines in Isaurien stationierten Vaters dort aufgewachsen, hatte dann in Armenien militärische Karriere gemacht und kannte aus diesem Grund Terrain und Verhältnisse in beiden Provinzen. ${ }^{138}$ Nach Isaurien kehrte er mit den beiden pseudocomitatensischen armenischen Legionen Armeniaca I und wahrscheinlich auch II zurück. Sein genauer militärischer Rang ist nicht bezeugt - er kann als einfacher comes rei militaris nach lsaurien gekommen sein, eventuell sogar für dieses Amt zum comes Isauriae oder gar zum magister militum praesentalis ernannt worden sein. ${ }^{139}$ Arbazacius jagte im Jahr 404 die Räuber aus der pamphylischen Ebene in die Berge und begann damit, ihre Dörfer zu zerstören. Damit verhinderte er nicht nur ein weiteres Ausgreifen der Raubzüge in die pamphy-

136 Hier. ep. 114, 1: ... multa in medio impedimenta fecerunt: Isaurorum repentina eruptio: Phoenicis Galileaeque vastitas: terror Palestinae, praecipue urbs Hierosolymae: et nequaquam librorum sed murorum extmictio. Ad hoc asperitas hiemis, fames intolerabilis, nobis praesertim, quibus multorum fratrum cura inposita est. LABOURT Hier. (1958) 166 will hier eine Verwechslung mit den Tribigild-Goten nicht ausschließen, was angesichts der übrigen Quellenzeugnisse unwahrscheinlich ist.

137 Eun. frg. 71, 1 = Suda A 3752; Zos. V 25, 2; PLRE II 127f. s.n. 'Arbazacius 1'; Woods (1998) passim.

138 CTh VII 22, 1-12; P.Abinn. 19; Sulp. Sev. V. Mart. 2 zu dieser Praxis. Woods (1998) 113 löst den bei Eun. frg. 71, 1 gezeichneten Widerspruch 'A $\beta \alpha \zeta \alpha$ ' $\kappa$ Los, "I $\sigma \alpha \cup \rho \circ \varsigma$ [...] $\hat{\eta} v$

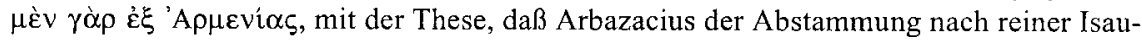
rier gewesen sei, der nur seine Jugend in Armenien verbracht habe, gefolgt von WILLIAMS / FrIELL (1999) 28: Arbazacius sei „himself distantly Isaurian in origin“. Doch diese Behauptung läßt sich mit gleichem Recht umdrehen und ist angesichts seines armenischen Namens eher unwahrscheinlich, vgl. JusTI (1895) 21. 89. Nach PLRE II 127f. s.n. 'Arbazacius 1' u. BlockLEY (1983) 148, Anm. 168 kann sein Epitheton "I $\sigma \alpha v \rho \circ$ ebenso gut durch sein Wirken in dieser Gegend geprägt worden sein, nach Art eines nom de guerre.

139 Marc. com. s.a. 405 bezeichnet ihn als legatus: Isauri per montem Tauri discursantes ingens dispendium rei publicae importarunt: quibus Narbazaicus legatus maius continuo rependit incommodum. Zos. V 25 nennt ihn zwar schlicht $\sigma \tau \rho \alpha \tau \eta \gamma o ́ s$ berichtet aber im folgenden von seinen guten Beziehungen zum Kaiserhaus, was auf einen hohen Rang schließen läßt. Nach RidLEY (1970) 93, Nr. 21 war er magister militum. Die von WoodS verfochtene Möglichkeit, daß Arbazacius zunächst comes rei militaris der beiden armenischen Legionen gewesen, dann aber als comes Isauriae nach Isaurien zurückkehrte, kann nicht ausgeschlossen werden. - Der von DEMANDT (1970) 736 in Synes. ep. 134 (aus dem Jahr 396) genannte 'A $\tau \tau \alpha \beta \alpha ́ \zeta \alpha \kappa \circ \varsigma \delta v v \alpha \sigma \tau \varepsilon v \sigma \alpha \alpha \varsigma$ ist nicht mit dem Militär, sondern mit dem praeses Libyae Superioris zu identifizieren, vgl. PLRE II 154 s.n. 'Artabazaces'. 
lische und lykische Ebene, sondern zwang sie auch, nach Osten auszuweichen, um der physischen Vernichtung zu entgehen. Die ausgedehnten Plünderungen im östlichen Mittelmeerraum können also als eine direkte Folge der Militäraktionen des Arbazacius bezeichnet werden. Langfristig konnte es durch bloßen militärischen Terror nicht gelingen, die Region zu befrieden. Zudem führte er die Kampagne nicht zu Ende, denn er ließ sich offenbar durch isaurische Bestechungsgelder von einem weiteren Vorgehen abbringen. ${ }^{140}$ Aus diesem Grunde folgte zwar eine Vorladung in den Kaiserpalast mit anschließendem Gerichtsverfahren. Laut Zosimus soll er jedoch einen Teil seines Geldes der Kaiserin zugeschanzt haben, worauf der Prozeß niedergeschlagen wurde. ${ }^{141}$ Die Ungenauigkeit des Zosimus zeigt sich in dem folgenden Satz: Die Isaurier hätten ,ihre Unternehmungen noch insgeheim fortgesetzt, ohne es aber noch zu einer offenen Empörung gegen die Nachbarprovinzen kommen zu lassen. " ${ }^{\star 142}$ Die $\mathrm{ab}$ diesem Zeitpunkt beginnenden ausgedehnten Scharmützel und Plünderungen im östlichen Mittelmeerraum erwähnt Zosimus in seiner bis zum Jahr 410 reichenden Geschichte nicht - wohl weil die Geschichte des Eunap nur bis 404 reichte. ${ }^{143}$ Diese Plünderungen führten die isaurischen Banden bis nach Palästina, an die Schwarzmeerküste und an die persische Grenze. Von dort aus kehrten sie um, plünderten Pamphylien, Lykien, Cypern, Lykaonien und Pisidien, Kappadokien und von dort aus hinauf bis zum Schwarzen Meer. Theodoret beschreibt in der Vita des Mönchs Theodosius eine Begegnung mit isaurischen Banditen und legt damit nahe, daß sie auch dessen Aufenthaltsort, die Gegend der Hauptstadt der Diözese Oriens selbst, Antiochia am Orontes heimsuchten. ${ }^{144}$ Zwei Bischöfe nahmen sie als Geiseln und ließen sie für ein Lösegeld von 40000 Goldstücken frei. ${ }^{145}$ In Antiochia plünderte eine Bande kurz darauf die Akropolis und massakrierte eine große Zahl von Frauen, Kindern und

140 MacMullen (1988) 183 übernimmt die Wertung von Eunap und Zosimus über Arbazacius: statt die Isaurier vollständig zu erobern, habe er sich damit begnügt, die Beute der ersten Gefechte zu verprassen.

141 Zos. V 25, 1-4. Marc. Com. s.a. 405 datiert ein Jahr zu spät, da Zos. V 25, 4 schreibt, er habe seine Beute der Kaiserin Eudoxia zur Verfügung gestellt und dadurch eine gerichtliche Verfolgung vermieden. Diese hatte jedoch am 6. Oktober 404 eine Fehlgeburt erlitten, woran sie gestorben war. Die Chronologie ist insgesamt unsicher, vgl. REBENICH Zos. (1990) 370, Anm. 48. 373, Anm. 57f.

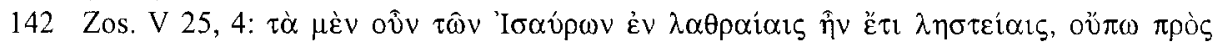

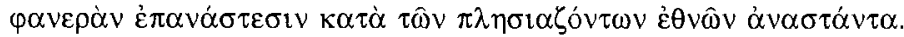

143 Phot. Bibl. 77; vgl. Paschoud Zos. (1986) IIIl 188 ff. 190: „La guerre isaurienne se prolongea en tout cas jusq'en $407^{\prime \prime}$.

144 Theod. h. r. X 5; Rekonstruktion der Plünderungszüge bei RougÉ (1966).

145 Theod. h. $r, \mathrm{X} 6$. 
Geistlichen. ${ }^{146}$ Theodoret predigt in der Vita des Jakob gegen die offensichtlich grassierende Furcht in diesen Landesteilen vor den isaurischen Banditen. ${ }^{147}$

Die zwischen Antiochia und Isaurien liegende östliche Taurusregion war zu dieser Zeit gänzlich unsicher geworden: Der mittlerweile abgesetzte Konstantinopler Bischof Johannes Chrysostomus schrieb in seinen „Briefen aus dem Exil“, das er ab Juni 404 (dem Jahr von Arbazacius' Isauriersieg) in Cucusus in der Armenia Secunda und - nach gefährlicher Reise durch die unsichere Landschaft - bis zu seinem Tod im Jahr 407 in Caesarea in der Cappadocia verbrachte, daß die plündernden Isaurier ihm auf jeder seiner Reisestationen zuvorgekommen seien. In Cucusus waren die Bewohner bei seiner Ankunft in Furcht vor den Isauriern. ${ }^{148}$ Ende 404 machten sie zunächst die Gegend um Caesarea unsicher; das Militär mußte durch Milizen verstärkt werden. ${ }^{149}$ Doch mit Einbruch des Winters waren die Isaurier plötzlich wieder nach Hause verschwunden, Johannes rechnete mit ihrem neuerlichen Erscheinen nicht vor dem nächsten Pfingsten. ${ }^{150}$ Der oftmals vom Fieber geplagte Exulant begab sich in den folgenden Jahren, wenn die Gegend durch isaurische Räuberbanden gefährdet wurde, in die befestigte Stadt Anabissus (heute Yarpuz). Doch auch dieses Kastell war nicht sicher: Einmal versuchte eine Horde von 300 Isauriern, allerdings erfolglos, das Kastell bei Nacht zu stürmen. ${ }^{151}$ Dennoch konnten sie eine Anzahl von Gefangenen nehmen, die Johannes zurückkaufte. ${ }^{152}$

Die isaurischen Banden konnten sich nach diesem Vorfall noch ungefähr zwei weitere Jahre lang in die Armenia secunda einfallen; zum Jahreswechsel 406/407

146 Theod. $h$. $r$. XII 6; DEMANDT (1989) 313; die im Text genannte „Akropolis“ läßt sich nicht nachweisen, vgl. Downey (1961) 439; CANIVET / Leroy-MolingheN Theodoret (1977) 469.

147 Theod. h. r. XXI 27.

148 Joh. Chrysost. ep. ad Olympiadem VI (September 404). Zu den Hintergründen seines Exils vgl. Zos. V 23; Soc. h.e. VI 15 ff;; Soz. h.e. VIII 16 ff; Pallad. Dial.(passim); Heinrich LieTzMANN, s.V. 'Ioannes 55', RE IX 2 (1916) 1811-1828; SEECK (1921) V $335-$ 370; SteIn (1928) 373 ff.; Christoph BAuR, Johannes Chrysostomus und seine Zeit, 2 Bde., München 1929/1930; Demougeot (1951) 296-337; Malingrey Joh. Chrys. ('1968) 12-53; Jean-Marie Leroux, TRE 17 (1988) 118-127; ReBenich Zos. (1990) 370f., Anm. 49; Karl-Heinz Uthemann, BBKL III (1992) 305-326. 318; Kelly (1995) 253-260; Peter Klasvogt, LThK 5 (1996) 889-892; TiersCH (2002) 397-410.

149 Joh. Chrysost. ep. ad Olympiadem IX; ibid. 2 c (Z. 29 ff.) schildert er, daß eine Horde plündernder Mönche, die sogar Soldaten in die Flucht schlug, eine nicht geringere Bedrohung als die Isaurier darstellte.

150 Joh. Chrysost. ep. ad Olympiadem IX 4 c (Z. 23-30).

151 Joh. Chrysost. ep. 135. 142.

152 Soz. h.e. VIII 27. 
waren sie keine Bedrohung mehr für Johannes. ${ }^{153}$ Das Militär hatte schließlich gesiegt, die Räuber wurden der Justiz zugeführt. Ihre große Zahl verdeutlicht das Gesetz des Arcadius vom 27. April 408. Entgegen der üblichen Praxis der Osteramnestien sollten Verhöre und Prozesse nicht einmal in der Fastenzeit und zu Ostern gegen gefaßte isaurische Räuber ausgesetzt werden. ${ }^{154}$

Andererseits schildern die gegen Mitte des 5. Jahrhunderts entstandenen Miracula der heiligen Thekla auch für die folgenden Jahrzehnte eine generelle Unsicherheit im gesamten Raum, die trotz des Sieges über die großen isaurischen Räuberbanden um die Jahrhundertwende noch vorherrschte. Selbst Bewaffnete konnten sich auf den Straßen nicht sicher fühlen: Der Autor der Miracula vermerkt es als Besonderheit, daß der kaiserliche Gesandte Ambrosius, ein Soldat der scholae palatinae, trotz der Gefährlichkeit der Straßen unbehelligt nach Konstantinopel gelangte. ${ }^{155}$

Mit den großen Aufständen, die zeitlich mit existenzbedrohenden Auseinandersetzungen mit den Goten - die Schlacht bei Adrianopel 378 und die Revolten von Tribigild und Gainas 399/400 - zusammenfielen, hatten die Isaurier ihren Ruf, den äußeren Reichsfeinden ebenbürtig zu sein, für die folgenden Jahrhunderte zementiert. Welche Gründe dies im einzelnen hatte, soll im folgenden Kapitel dargelegt werden.

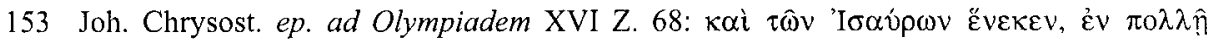
$\kappa \alpha \theta \varepsilon \sigma \tau \eta \dot{\kappa} \alpha \mu \varepsilon v \dot{\alpha} \sigma \varphi \alpha \lambda \varepsilon i \alpha \underline{\alpha}$. XVII Z. 2.

$154 C T h$ IX 35, 7 = CJ III 12, 8: ut in Isaurorum latronum questionibus nullum quadragesimae nec venerabilem pascharum diem existiment [scil. provinciarum iudices] excipiendum; vgl. DEMANDT (1989) 313. Erste Osteramnestien finden sich unter Valentinian und Valens: CTh IX 38, 3f, mit der Constitutio Sirmondiana waren sie unter Theodosius im Jahr 386 wahrscheinlich zur festen Einrichtung geworden; vgl. Wolfgang WALDSTEIN, Untersuchungen zum römischen Begnadigungsrecht (= Commentationes Aenipontanae 18), Innsbruck 1964; Bernhard RasPELS, 'Der Einfluß des Christentums auf die Gesetze zum Gefängniswesen' in: ZKG 102 (1991), 289-306, 295 u. Anm. 38.

155 Mir. 16 mit Dagron (1978) 333, Anm. 1. 